\title{
In vivo screening reveals interactions between Drosophila Manf and genes involved in the mitochondria and the ubiquinone synthesis pathway
}

\author{
Riitta Lindström ${ }^{1,4^{*}}$ (D) Päivi Lindholm², Mari Palgi ${ }^{3}$, Mart Saarma ${ }^{2}$ and Tapio I. Heino ${ }^{1^{*}}$
}

\begin{abstract}
Background: Mesencephalic Astrocyte-derived Neurotrophic Factor (MANF) and Cerebral Dopamine Neurotrophic Factor (CDNF) form an evolutionarily conserved family of neurotrophic factors. Orthologues for MANF/CDNF are the only neurotrophic factors as yet identified in invertebrates with conserved amino acid sequence. Previous studies indicate that mammalian MANF and CDNF support and protect brain dopaminergic system in non-cell-autonomous manner. However, MANF has also been shown to function intracellularly in the endoplasmic reticulum. To date, the knowledge on the interacting partners of MANF/CDNF and signaling pathways they activate is rudimentary. Here, we have employed the Drosophila genetics to screen for potential interaction partners of Drosophila Manf (DmManf) in vivo.

Results: We first show that DmManf plays a role in the development of Drosophila wing. We exploited this function by using Drosophila UAS-RNAi lines and discovered novel genetic interactions of DmManf with genes known to function in the mitochondria. We also found evidence of an interaction between DmManf and the Drosophila homologue encoding Ku70, the closest structural homologue of SAP domain of mammalian MANF.

Conclusions: In addition to the previously known functions of MANF/CDNF protein family, DmManf also interacts with mitochondria-related genes. Our data supports the functional importance of these evolutionarily significant proteins and provides new insights for the future studies.
\end{abstract}

Keywords: MANF, CDNF, Genetic screen, Mitochondria, Ubiquinone

\section{Background}

An evolutionarily conserved protein family, MANF/ CDNF family, is the most recently discovered family of neurotrophic factors (NTFs) [1-4]. Typically of NTFs, MANF and CDNF are small secreted molecules that support the survival of neurons [1, 2]. Mammalian MANF and CDNF support the brain dopaminergic system in rodent models of Parkinson's disease (PD) in vivo $[2,5,6]$. MANF has been shown to protect neurons and cardiomyocytes against ischemic injury in extracellular manner [7, 8]. Additionally, MANF is required for the proliferation and survival of the pancreatic $\beta$-cells [9].

\footnotetext{
* Correspondence: riitta.lindstrom@helsinki.fi; tapio.heino@helsinki.fi ${ }^{1}$ Department of Biosciences, University of Helsinki, Fl-00014 Helsinki, Finland

Full list of author information is available at the end of the article
}

Orthologues for MANF/CDNF are the only neurotrophic factors as yet identified in invertebrates with conserved amino acid sequence $[1,3]$. The invertebrate homologues show higher similarity to mammalian MANF than CDNF $[2,3]$. However, both human MANF and CDNF are functional orthologues of Drosophila Manf (DmManf) [3, 10]. In Drosophila, glial-derived DmManf is necessary for maintaining the neurites of embryonic and larval dopaminergic neurons that do not express DmManf. This demonstrates that the extracellular trophic function for dopaminergic system is conserved [3].

The knowledge on the molecular interactions of MANF/CDNF family proteins remains limited. Also the receptor for MANF/CDNF proteins is not known. Intracellularly, mammalian MANF has been shown to bind 
GRP78/BiP (Glucose-regulated protein 78/Binding immunoglobulin protein), the major ER chaperone, in $\mathrm{Ca}^{2+}$-dependent manner [8]. There is also experimental evidence suggesting that MANF interacts with KDELRs, KDEL (Lys-Asp-Glu-Leu) endoplasmic reticulum protein retention receptors [11]. Furthermore, a recent study suggests that MANF interacts with a member of ER-associated reticulon protein family [12]. Our previous study shows a genetic interaction between DmManf and Drosophila homologues of GRP78, PERK (PRKR-like endoplasmic reticulum kinase, one of the ER stress sensor proteins) and Xbp1 (X-box Binding Protein-1, a transcription factor mainly mediating ER stress response activated gene expression) [13]. Additionally, our earlier microarray analysis suggests that DmManf has a role in Drosophila ER stress response [14]. MANF is localized to ER [14-17] and the retention is mediated through the non-classical but evolutionarily conserved ER retention signal sequence, RTDL in human and RSEL in Drosophila $[8,10,17]$. Furthermore, the expression of Manf mRNA is induced in response to ER stress [13, 15, 17-20]. In addition to GRP78, co-immunoprecipitation studies have revealed that MANF (also known as Armet) interacts with a mutant form of an extracellular matrix protein matrilin 3 [21].

Both mammalian and Drosophila MANF have been shown to hold intracellular cytoprotective function against Bax (BCL-2 associated X) -dependent cell death in vitro $[10,22]$. The $C$-terminal domain of MANF shows high structural homology to SAP (SAF-A/B, Acinus and PIAS) domain of Ku70 (Ku autoantigen p70 subunit), an inhibitor of Bax-mediated apoptosis [23], and it is alone capable of protecting neurons from induced apoptosis in vitro [10,22].

MANF and CDNF have been suggested to be involved in inflammatory responses [24-28]. The main mediator of proinflammatory response, NF- $\mathrm{kB}$ (nuclear factor kappa-light-chain-enhancer of activated B cells), is also regulated by unfolded protein response, a cellular process activated by ER stress (reviewed e.g. in [29]). In a recent study MANF was found to bind the p65 subunit of NF- $\mathrm{kB}$ via the C-terminal SAP-domain in vitro [28]. Upon inflammation, MANF localized to nucleus and was suggested to suppress the expression of NF- $\mathrm{kB}$ targets by binding to DNA binding domain of p65 as well as to adjacent enhancer regions of target genes [28]. Interestingly, recent study demonstrated that MANF has a conserved immune modulatory function in both Drosophila and mouse promoting tissue repair and regeneration in retina [30].

In this work we used RNA interference (RNAi) approach in UAS/GAL4 in vivo system to study interacting partners of DmManf in Drosophila model. In the binary UAS/GAL4 system, GAL4 lines with various expression patterns are used for tissue-specific expression of UAS (upstream activation sequence) -transgenes [31]. RNAi where double stranded RNA (dsRNA) induces the degradation of targeted mRNA [32] is commonly used for gene silencing. Transgenic genome-wide Drosophila RNAi libraries have been established [33] (http:// www.shigen.nig.ac.jp/fly/nigfly/) by introducing dsRNAs under UAS promotor. Crossing these flies with different GAL4 driver lines enables tissue-specific target gene inactivation. Expression of other UAS constructs or markers (e.g. GFP) can be simultaneously activated in the same GAL4 expression pattern. In this study, we used UAS-DmManf-RNAi construct for targeted knockdown of DmManf and performed a partial, unbiased screen of RNAi libraries in vivo to discover novel interacting partners for DmManf. Here we demonstrate genetic interactions between DmManf and genes with mitochondrial function.

\section{Results}

Silencing of DmManf by UAS-DmManf-RNAi is effective and specific in vivo

Homozygous DmManf mutants die at early developmental stage [3]. To study the role of DmManf during later stages of development we used the UAS/GAL4 system for tissuespecific knockdown of DmManf [31, 33]. Three UASDmManf-RNAi fly stocks were obtained from Vienna Drosophila RNAi Center (VDRC) (A in Additional file 1). All transformant lines showed similar phenotypes with different GAL4 drivers (B in Additional file 1), and the transformant line 12835 with construct ID 4793 was used in further experiments.

The ubiquitous knockdown of DmManf with tubGAL4 and da-GAL4 drivers in wild type background showed lethal phenotype at larval to pupal stage (Fig. $1 \mathrm{a}-\mathrm{b}$ and data not shown). The knockdown efficiency of DmManf expression was verified at both mRNA and protein level by quantitative RT-PCR (qPCR) and Western blot analyses, respectively (Fig. 1c-d). When the ubiquitous knockdown of DmManf was performed in heterozygous DmManf $f^{196}$ mutant background with already decreased DmManf protein level, the lethality was observed at early larval stage (Fig. 1a) resembling the phenotype of homozygous DmManf ${ }^{196}$ mutants [3].

According to information provided by VDRC, there are no predicted off-targets for DmManf-RNAi construct ID 4793. To verify the specificity of DmManfRNAi, DmManf was simultaneously overexpressed (by UAS-DmManf ${ }^{\mathrm{L}}$ ) and knocked down (by UASDmManf-RNAi) with ubiquitous tub-GAL4 driver. The overexpression of DmManf rescued the pupal lethality phenotype of ubiquitous DmManf knockdown flies into adulthood (Fig. 1a-b). We also used overexpression of the UAS constructs encoding transcripts for DmManf 


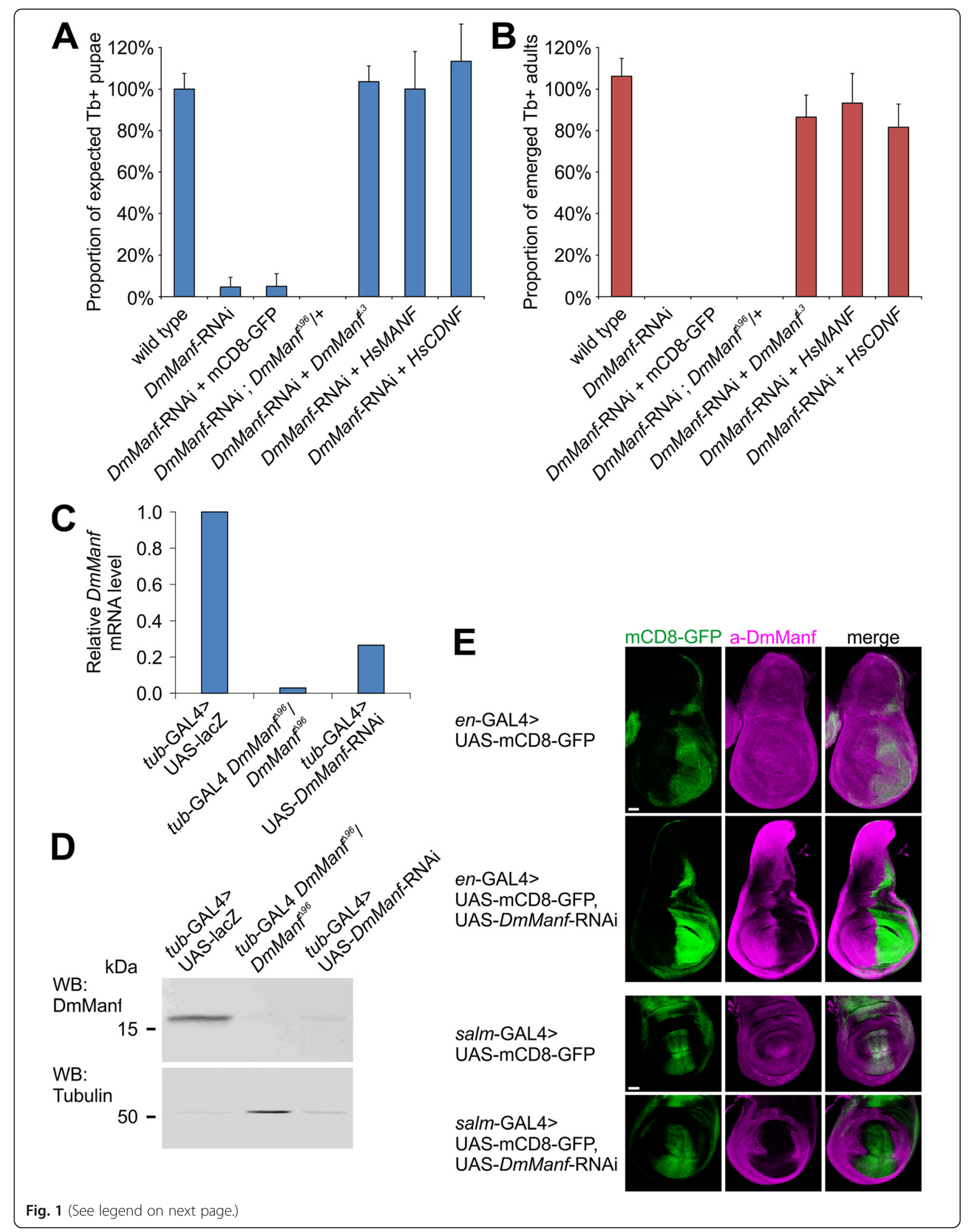


(See figure on previous page.)

Fig. 1 Knockdown of DmManf by UAS-DmManf-RNAi is effective and specific. a-b Quantification of pupae (a) and adults (b) of ubiquitous knockdown of DmManf with tub-GAL4 driver. Ubiquitous knockdown of DmManf was lethal at larval stage with few escapers to pupal stage. UAS-mCD8GFP was used as a dose control for UAS/GAL4 binary expression system, tub-GAL4/+ as a wild type control. Amount of pupae and adults analysed are presented in Additional file 8. Proportion of $\mathrm{Tb}^{+}$pupae was normalized to experimentally determined proportion of $\mathrm{Tb}^{+}$pupae (see Additional file 8 , wild type and wild type/SM6-TM6). c-d Quantitative RT-PCR (c) and Western blot (d) analyses revealed that ubiquitous knockdown of DmManf resulted in robustly reduced DmManf mRNA and protein levels in larvae collected 50-54 h after egg laying. Homozygous DmMant ${ }^{96}$ mutant showed diminished level of maternally contributed DmManf mRNA and protein. tub-GAL4 > UAS-lacZ flies were used as a wild type control. DmManf mRNA levels were normalized to wild type. Alpha-tubulin was used as a loading control for Western blot analysis. e Knockdown of DmManf with en-GAL4 and salm-GAL4 drivers showed a loss of DmManf immunoreactivity (magenta) in GAL4-expressing pattern. UAS-mCD8-GFP was used to detect GAL4 expression (green). Single laser confocal sections. Scale bar $50 \mu \mathrm{m}$

human (Hs) orthologues, HsMANF and $H s C D N F$, which share less homology with the DmManf-RNAi construct (C in Additional file 1) than DmManf. Both HsMANF and $H s C D N F$ rescued the pupal lethality observed in DmManf ubiquitous knockdown flies (Fig. 1a-b). When two UAS constructs are used in the same fly, GAL4 protein supply is shared by the two promotor regions and might lead to decreased expression of UAS targets. In the case of UAS-RNAi lines, this dose effect could compromise the knockdown efficiency. To confirm that the rescue of DmManf knockdown phenotype was not due to inefficient knockdown of DmManf, we used UAS-DmManf-RNAi; UAS-mCD8-GFP line as a dose control for UAS/GAL4 binary expression system. Ubiquitous knockdown of DmManf by UAS-DmManf-RNAi; UAS-mCD8-GFP with tub-GAL4 showed similar proportion of expected pupae to UAS-DmManf-RNAi alone (Fig. 1a-b).

\section{Wing-specific knockdown of DmManf drastically alters wing morphology and increases cell proliferation}

DmManf is ubiquitously expressed in 3rd instar larval wing disc [10]. To verify the efficiency of tissue-specific silencing of DmManf, we used salm-GAL4 and en-GAL4 to knock down DmManf and simultaneously express UAS-mCD8-GFP to visualize the GAL4 expression pattern in the larval wing disc. The loss of DmManf immunoreactivity was detected exactly according to salm-GAL4 and en-GAL4 expression pattern in the wing disc (Fig. 1e) further demonstrating that the knockdown of DmManf was efficient at protein level. Interestingly, in DmManf knockdown wing discs we detected mild but clear increase of DmManf immunoreactivity in regions next to the GAL4 expressing area (Fig. 1e). This might indicate a compensatory regulation of DmManf expression in response to the partial loss of DmManf in the wing disc.

Interestingly, we observed two different wing phenotypes when various drivers with GAL4 expression in the wing were used to knock down DmManf in wild type background. The knockdown with MS1096-GAL4, A9-GAL4 and Ser-GAL4 drivers showed a threedimensional "bent-up" wing phenotype (b3 in Fig. 2a and data not shown) while knockdown with 69B-GAL4 revealed blistered wing phenotype (Fig. 2b). The wing phenotype was observed with full penetrance in males of DmManf knockdown with MS1096-GAL4 (Table 1). To verify further the specificity of DmManf knockdown, we repeated the experiments in heterozygous DmManf ${ }^{\Delta 96}$ mutant background. With 69B-GAL4, the knockdown of DmManf in heterozygous DmManf ${ }^{196}$ mutant background resulted in lethality. When MS1096-GAL4 driver was used to knock down DmManf in heterozygous DmManf ${ }^{96}$ mutant background, the wing phenotype was stronger as compared to DmManf knockdown in wild type background, especially in males in which the wing blade was totally lost (b2 and b4 in Fig. 2a). In comparison to females, the more severe knockdown wing phenotype observed in males was likely due to the dosage compensation of X-chromosomal GAL4 insertion in MS1096-GAL4 driver line.

Simultaneous expression of UAS-DmManf ${ }^{\mathrm{L} 3}$ and UAS-DmManf-RNAi with MS1096-GAL4 returned the wings back to wild type (c1-c2 in Fig. 2a) indicating that overexpression of DmManf could rescue the wing phenotype. Co-expression of UAS-HsMANF or UAS$H s C D N F$ together with UAS-DmManf-RNAi by MS1096-GAL4 also rescued the wing phenotype (d1-d4 and e1-e4 in Fig. 2a). This further demonstrated that the knockdown of DmManf by UAS-DmManf-RNAi construct was specific to the DmManf mRNA only and suggests that DmManf plays an important role during wing development.

To investigate whether DmManf is involved in regulation of cell proliferation in vivo, we used the wing-specific knockdown of DmManf with MS1096-GAL4. Wing discs of third instar male larvae were stained for a mitotic marker phosphorylated Histone-3 (pHis3) [34]. We found that the number of pHis3 positive cells within GFP-expressing wing area was significantly increased when DmManf was knocked down in heterozygous DmManf ${ }^{496}$ mutant background in comparison to heterozygous DmManf ${ }^{196}$ mutant alone (Fig. 3a-b). In addition, we used UAS-S/G2/M-Green transgenic flies based on the Fucci (fluorescent, ubiquitination-based 


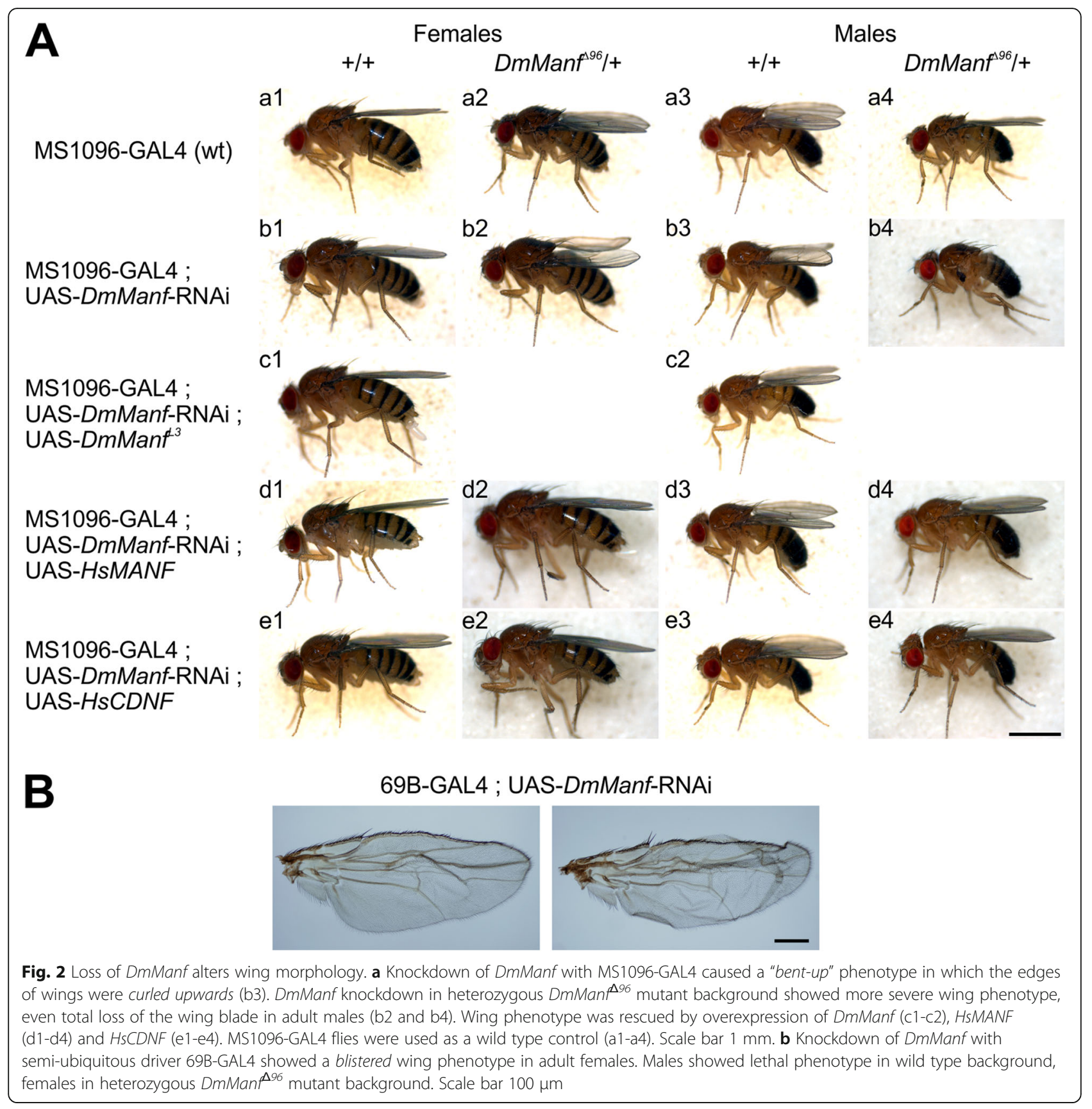

cell cycle indicator) model $[35,36]$. act-His2AvRFP transgene was used to visualize nuclei of all cells. We fixed 3rd instar wing discs of male larvae and detected increased proportion of $\mathrm{S} / \mathrm{G} 2 / \mathrm{M}$ cells in DmManf knockdown with MS1096-GAL4 driver in comparison to wild type (Fig. $3 \mathrm{c}-\mathrm{d}$ ). This further indicates that the loss of DmManf affects cell cycle events.

\section{Screening of DmManf interaction partners}

The observed wing phenotype allowed us to perform a straightforward genetic screen to identify novel genetic interactions of DmManf. Overall scheme of our four- staged RNAi screen is presented in Additional file 2. We screened approximately 2800 RNAi lines (representing about $20 \%$ of fly genome) from VDRC and National Institute of Genetics (NIG, Japan) collections. The RNAi lines were selected randomly to perform unbiased screening for the interacting partners. The primary screen was conducted with MS1096-GAL4 driver (E. Vridolin and O. Shimmi, unpublished data) and RNAi lines with similar wing phenotype to DmManf-RNAi were selected for secondary screening. MS1096-GAL4 is expressed in the pouch area of the wing disc ( $B$ in Additional File 2, [37-39]), but we also detected a weak 
Table 1 Penetrance of the wing phenotype in adult flies of DmManf knockdown with MS1096-GAL4

\begin{tabular}{lll}
\hline Genotype & $\begin{array}{l}\text { Proportion of males } \\
\text { with phenotype }\end{array}$ & $\begin{array}{l}\text { Proportion of females } \\
\text { with phenotype }\end{array}$ \\
\hline MS1096-GAL4; & $140 / 140$ & $102 / 176$ \\
UAS-DmManf-RNAi & & \\
MS1096-GAL4; & $0 / 220$ & $0 / 218$ \\
UAS-mCD8-GFP & & \\
\hline
\end{tabular}

expression pattern in central nervous system (CNS) (C in Additional File 2). For more efficient screening, we focused on the wing phenotypes only. The secondary screen was performed for approximately 400 RNAi lines with two GAL4 drivers, MS1096-GAL4 and semi-ubiquitous 69B-GAL4, in both wild type and heterozygous DmManf ${ }^{196}$ mutant backgrounds to observe whether the phenotype caused by silencing of a particular gene would be altered by decreased DmManf expression level [3]. 69B-GAL4 lacks expression in the fat body, gastric caeca and muscles, and its expression patterns in the CNS and cuticle are limited [10, 14]. In addition to its semi-ubiquitous expression pattern, we chose 69B-GAL4 driver because DmManf ${ }^{966}$ mutant lethality is fully rescued by ectopic expression of UAS-DmManf with 69B-GAL4 [3]. About 80 RNAi lines showed altered phenotype between wild type and heterozygous DmManf $\Delta 96^{\Delta 96}$ mutant background and were selected for tertiary screening. In tertiary screening, we used overexpression of DmManf together with knockdown of candidate genes with 69B-GAL4 and MS1096-GAL4 drivers to study whether the increased level of DmManf would affect the phenotypes caused by silencing of the candidate genes. The overexpression of DmManf with 69B-GAL4 or MS1096-GAL4 alone showed no detectable phenotype in adults ( $\mathrm{D}$ in Additional file 2). Based on secondary and tertiary screening, we selected 21 genes as candidate interacting partners of DmManf (Fig. 4). Examples of candidate gene knockdown experiments are presented in Fig. 5a-b and Additional file 3.

The putative interactions between DmManf and the 21 candidate genes were further analysed by ubiquitous knockdown of the candidate genes (stage 4 in Additional file 2, B-C in Additional file 3, Additional file 4). We used tub-GAL4 driver and compared pupal and adult viability between wild type background and with DmManf overexpression to see whether overexpression of DmManf would affect the phenotypes caused by ubiquitous knockdown of the candidate genes. For example, simultaneous overexpression of DmManf significantly decreased the pupal viability for ubiquitous knockdown of CSN3 (COP9 complex homolog subunit 3) encoding a protein involved in regulation of the ubiquitin conjugation pathway (B-C in Additional file 3). Overexpression of DmManf alone with tub-GAL4 in wild type background showed no obvious phenotype or affected fly viability [13].

\section{Gene ontology terms of potential DmManf interacting partners}

We analysed the Gene Ontology (GO) terms of the 21 candidate genes (Table 2). Four of the candidate genes were unannotated and excluded from the analysis. Most enriched GO terms were related to ubiquinone related processes (2 genes), mitochondrial cellular compartment (6 genes) and cellular metabolic process (13 genes). Alterations in genes annotated with GO terms related to metabolism were also observed in our previous microarray analysis [14]. The enrichment of mitochondrial genes was notable: $29 \%$ of our candidate genes were annotated with mitochondria as cellular compartment while according to FlyBase [40], only 5\% of all Drosophila genes have such annotation (http://gostat.wehi. edu.au/). Furthermore, three of our candidate genes (Ts, l(2)37Bb and CG9249) with unknown cellular compartment have human homologues which are annotated to mitochondria.

\section{DmManf partially localizes to mitochondria and genetically interacts with the ubiquinone synthesis pathway}

To examine further the interaction between DmManf and genes encoding mitochondrial proteins, we studied the subcellular localization of DmManf. According to our previous analyses, DmManf is localized to several cell compartments [14]. To detect mitochondria, we used sqh-EYFP-Mito transgenic fly line [41]. When the CNS of 3rd instar sqh-EYFP-Mito larvae were immunohistochemically stained with DmManf antibody, a partial co-localization with mitochondrial marker was detected (Fig. 5c and Additional file 5).

Two of our candidate genes were involved in the ubiquinone biosynthesis pathway, COQ7 (coenzyme Q7 homologue) and CG9249 (homologue of coenzyme Q3, COQ3) (Fig. 4 and Fig. 6). Ubiquinone is an electron carrier involved in respiratory electron transport chain localized to inner mitochondrial membrane (reviewed in $[42,43])$. The knockdown of COQ7 caused more severe phenotype with overexpression of DmManf than in wild type background when MS1096-GAL4, 69B-GAL4 or ubiquitous tub-GAL4 drivers were used. More specifically, the silencing of COQ7 with MS1096-GAL4 led to very mildly bent-up wings in wild type background (a1 and a3 in Fig. 5a). With simultaneous overexpression of DmManf this phenotype was stronger leading to small, wrinkled wings in adult males (a4 in Fig. 5a). The knockdown of COQ7 with 69B-GAL4 led to mildly uneven surface of the wing in wild type background but to a wrinkled wing phenotype when 


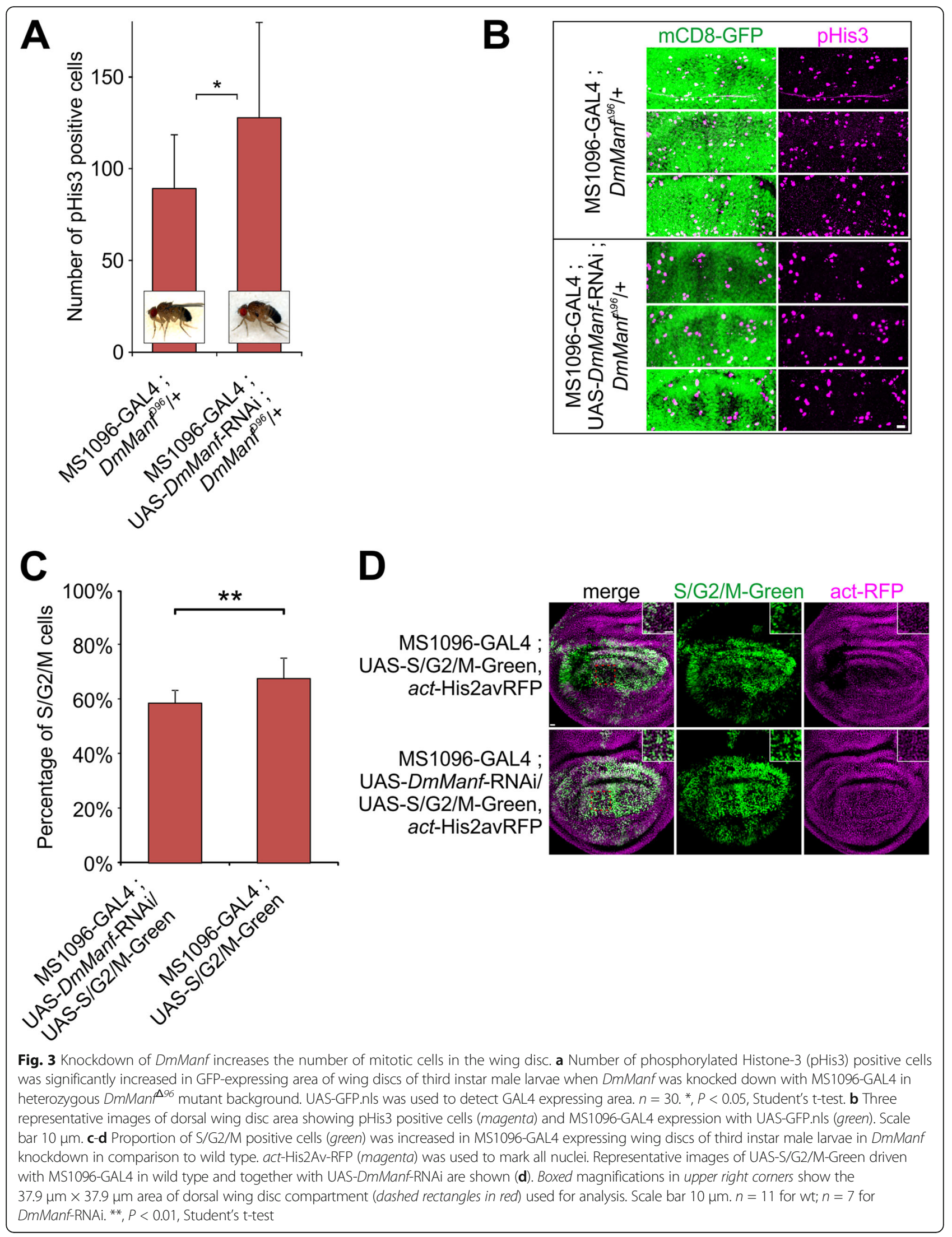




\begin{tabular}{|c|c|c|c|c|c|c|c|c|c|c|}
\hline \multirow[b]{2}{*}{ Gene name } & \multirow[b]{2}{*}{ Symbol } & \multirow[b]{2}{*}{ Human homologue(s) } & \multirow[b]{2}{*}{ 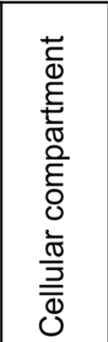 } & \multicolumn{4}{|c|}{ RNAi screen } & \multicolumn{3}{|c|}{ MAA } \\
\hline & & & & 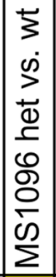 & $\begin{array}{l}\overrightarrow{3} \\
\dot{s} \\
w \\
0 \\
\mathscr{8} \\
8 \\
0 \\
\frac{D}{\Sigma} \\
\end{array}$ & 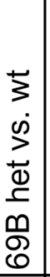 & 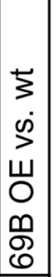 & 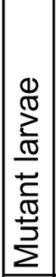 & $\begin{array}{l}\frac{1}{\pi} \\
\frac{\pi}{\sigma} \\
\frac{\sigma}{\omega} \\
\end{array}$ & 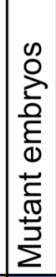 \\
\hline aurora borealis & bora & BORA & $\mathrm{Nu}, \mathrm{Cy}$ & & & & & & & \\
\hline betaTrypsin & betaTry & - & Ec & & & & & & & \\
\hline COP9 complex homolog subunit 3 & CSN3 & COPS3 & $\mathrm{Sg}$ & & & & & & & \\
\hline COQ7 & COQ7 & COQ7 & $\mathrm{Mi}$ & & & & & & & \\
\hline domeless & dome & - & $\mathrm{Me}$ & & & & & & & \\
\hline Heat shock protein 68 & Hsp68 & $\begin{array}{l}\text { HSPA2, }-6,-8,-1 A \text {, } \\
-1 \mathrm{~B},-1 \mathrm{~L}\end{array}$ & Mi & & & & & & & \\
\hline Inverted repeat-binding protein & $I r b p$ & XRCC6/Ku70 & $\mathrm{Nu}$ & & & & & & & \\
\hline lethal (2) 37Bb & I (2) $37 \mathrm{Bb}$ & FOXRED1 & nd & & & & & & & \\
\hline $\begin{array}{l}\text { NADH:ubiquinone reductase } \\
75 \mathrm{kD} \text { subunit precursor }\end{array}$ & ND75 & NDUFS1 & Mi & & & & & & & \\
\hline Phenylalanyl-tRNA synthetase & Aats-phe & FARS2 & $\mathrm{Mi}$ & & & & & & & \\
\hline polyA-binding protein & $p A B p$ & $\begin{array}{l}\text { PABC1, }-3,-4,5,-1 \mathrm{~L} \\
-1 \mathrm{~L} 2 \mathrm{~A},-1 \mathrm{~L} 2 \mathrm{~B}\end{array}$ & Cy,Sp & & & & & & & \\
\hline Serpin-27A & Spn27A & - & Ec & & & & & & & \\
\hline TBP-associated factor 5 & Taf5 & TAF5L & $\mathrm{Nu}$ & & & & & & & \\
\hline timeout & timeout & TIMELESS & nd & & & & & & & \\
\hline Translocase of outer membrane 20 & Tom20 & TOMM20, -20L & $\mathrm{Mi}$ & & & & & & & \\
\hline Ts & Ts & TYMS & nd & & & & & & & \\
\hline- & Fip1 & FIP1L1 & $\mathrm{Nu}$ & & & & & & & \\
\hline- & CG4707 & - & $\mathrm{Nu}$ & & & & & & & \\
\hline- & CG6455 & IMMT & $\mathrm{Mi}$ & & & & & & & \\
\hline- & Cdk12 & CDK9, -12, -13, & $\mathrm{Ch}$ & & & & & & & \\
\hline- & CG9249 & COQ3 & nd & & & & & & & \\
\hline
\end{tabular}

Color key / RNAi line screen:

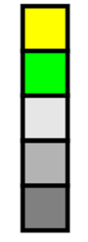

Stronger phenotype

Phenotype rescued

No phenotype in either genotypes

Phenotypes similar in both genotypes

Lethal phenotype in both genotypes
Color key / microarray data:

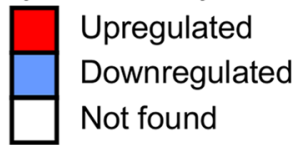

Fig. 4 Overview of candidate genes for interaction partners of DmManf based on RNAi screen. UAS-x-RNAi lines were crossed to MS1096-GAL4 and 69B-GAL4 driver lines in wild type, heterozygous DmManf ${ }^{\Delta 96}$ mutant and DmManf overexpression background. Observed phenotypes of knockdown flies in heterozygous DmMant ${ }^{\Delta 96}$ mutant background (het vs. wt) and DmManf overexpression background (OE vs. wt) were compared to phenotype of knockdown flies in wild type background. Yellow (stronger phenotype) and green (rescued phenotype) represent affected phenotypes. Light gray (no phenotype), gray (phenotype not affected) and dark gray (lethal phenotype) represent cases where heterozygous DmManf ${ }^{\Delta 96}$ mutant background or overexpression of DmManf did not affect the phenotype caused by knockdown of target gene. For a comparison, results from microarray analysis (MAA) [14] are presented; red and blue indicate up- and downregulation of target genes, respectively. Mutant larvae stands for zygotic DmManA ${ }^{\Delta 96}$ mutant larvae, OE larvae for 69B-GAL4 > UAS-DmMant3 larvae, and mutant embryos for maternal and zygotic DmMant ${ }^{\$ 96}$ mutant embryos. Cellular compartment: Ch, chromosome; Cy, cytoplasm; Ec, extracellular; Me, membrane; Mi, mitochondrion; Nu, nucleus; nd, no data available; Sg, signalosome; Sp, spliceosome. Het, heterozygous DmManf mutant; MAA, microarray analysis; OE, overexpression 
DmManf was overexpressed (c1-c4 in Fig. 5a). When COQ7 was ubiquitously knocked down with tubGAL4 in wild type background, lethality occurred during pupal stage and no adults emerged (B-C in Additional file 3). However, simultaneous overexpression of DmManf led to complete lethality at larval stage (B in Additional file 3). Ubiquitous knockdown of COQ7 with tub-GAL4 increased DmManf mRNA level determined by qPCR analysis (Fig. 6a).

Knockdown of COQ3/CG9249 with 69B-GAL4 and tub-GAL4 led to lethality at pupal and larval stages, respectively, and was not affected by either heterozygous DmManf ${ }^{96}$ mutant background or overexpression of DmManf (Fig. 4 and Additional file 4). When COQ3/ CG9249 was knocked down with MS1096-GAL4 in wild type background, a bent-up phenotype was observed in the adult wing (b1 and b3 in Fig. 5a, a1 and a3 in Fig. 5b). This phenotype was stronger leading to wrinkled wings when either simultaneous overexpression of DmManf (b1-b4 in Fig. 5a) or heterozygous DmManf ${ }^{196}$ mutant background (a1-a4 in Fig. 5b) was used. Ubiquitous knockdown of COQ3/CG9249 with tub-GAL4 showed a very slight increase in DmManf mRNA levels detected by qPCR analysis (Fig. 6a).

In Drosophila, only six genes are annotated to be involved in processes related to the ubiquinone synthesis pathway (GO:0006744 term in FlyBase, http:// flybase.org; Fig. 6b). We decided to study interaction between DmManf and the rest of the ubiquinone synthesis pathway genes, COQ2/CG9613, COQ6/CG7277, COQ9/CG30493, and COQ4/CG32174 (Fig. 6b-c). Wing-specific knockdown of any of these genes with MS1096-GAL4 showed no phenotype (Fig. 6c). Knockdown with semi-ubiquitous 69B-GAL4 driver showed either no phenotype (in the case of COQ6 and $C O Q 4)$ or led to lethality at pupal stage (COQ9 and COQ2) and observed phenotype was not altered by either heterozygous DmManf ${ }^{196}$ mutant background or simultaneous overexpression of DmManf (Fig. 6c). Simultaneous overexpression of DmManf did not affect the observed phenotype by ubiquitous knockdown of COQ6, COQ9 and COQ4 with tub-GAL4. However, we observed significantly decreased pupal viability when $C O Q 2$ was ubiquitously knocked down by tub-GAL4 in DmManf-overexpressing background compared to the wild type background ( $\mathrm{B}$ in Additional file 3; Additional file 4).

\section{DmManf genetically interacts with Irbp, the Drosophila homologue of Ku70}

Three dimensional structure of C-terminal domain of human MANF shows the highest similarity with the SAP domain of Ku70, an inhibitor of Bax-induced cell death $[22,23]$. Microinjected MANF encoding cDNA or protein has also been shown to protect cultured mouse superior cervical ganglion neurons from drug induced apoptosis $[10,22]$. In our genetic screen, we identified Irbp (Inverted repeat-binding protein), a homologue of mammalian Ku70, as a potential interacting partner of DmManf (Fig. 4). In adult male flies, knockdown of Irbp with MS1096-GAL4 driver in wild type background showed a wrinkled wing phenotype (a3 in Fig. 7a). When UAS-Irbp-RNAi was driven with MS1096-GAL4 in heterozygous DmManf $f^{196}$ mutant background, male flies showed a bent-up wing phenotype, clearly milder than in wild type background (a4 in Fig. 7a). In female flies, no alteration between wild type and heterozygous DmManf $f^{196} \mathrm{mu}$ tant background was detected (a1-a2 in Fig. 7a).

We noted that Irbp knockdown with MS1096-GAL4 resulted in smaller wing discs of 3rd instar wandering larvae in comparison to wild type (Fig. 7b). We quantified the width of the wing disc above the wing pouch area (indicated by red dashed line in Fig. 7b) and found that in the Irbp knockdown flies the width of the wing disc was significantly decreased in comparison to wild type (Fig. 7c). We also studied whether the heterozygous DmManf mutant background would affect the width of the wing disc in Irbp knockdown larvae with MS1096GAL4 and thus explain the altered wing phenotype of Irbp knockdown flies in this background. However, there was no difference in the width of the wing disc when Irbp was knocked down in wild type and heterozygous DmManf mutant backgrounds (Fig. 7c).

We observed lethality at pupal stage when Irbp was ubiquitously knocked down with tub-GAL4 (B in Additional file 3). Simultaneous overexpression of DmManf partially rescued this lethality to adulthood (B-C in Additional file 3) suggesting that DmManf and Irbp may act in the same pathway and have, to certain extent, redundant function.

To examine whether altered expression level of Irbp and DmManf affect each other, we collected embryos of DmManf knockdown as well as wandering 3rd instar larvae of Irbp knockdown and DmManf overexpression with semi-ubiquitous 69B-GAL driver and quantified Irbp and DmManf mRNA levels by qPCR analysis. In DmManf knockdown embryos, DmManf mRNA expression level was significantly decreased in comparison to the control (Fig. 7d). Interestingly, Irbp mRNA expression was also significantly decreased in DmManf knockdown embryos (Fig. 7d) indicating that loss of DmManf downregulated Irbp expression.

In Irbp knockdown larvae Irbp mRNA level was significantly decreased while DmManf mRNA expression was not altered (Fig. 7e). In DmManfoverexpressing larvae, the level of Irbp mRNA did not differ from the control genotype (Fig. 7e). When Irbp knockdown was done in wild type and DmManf- 


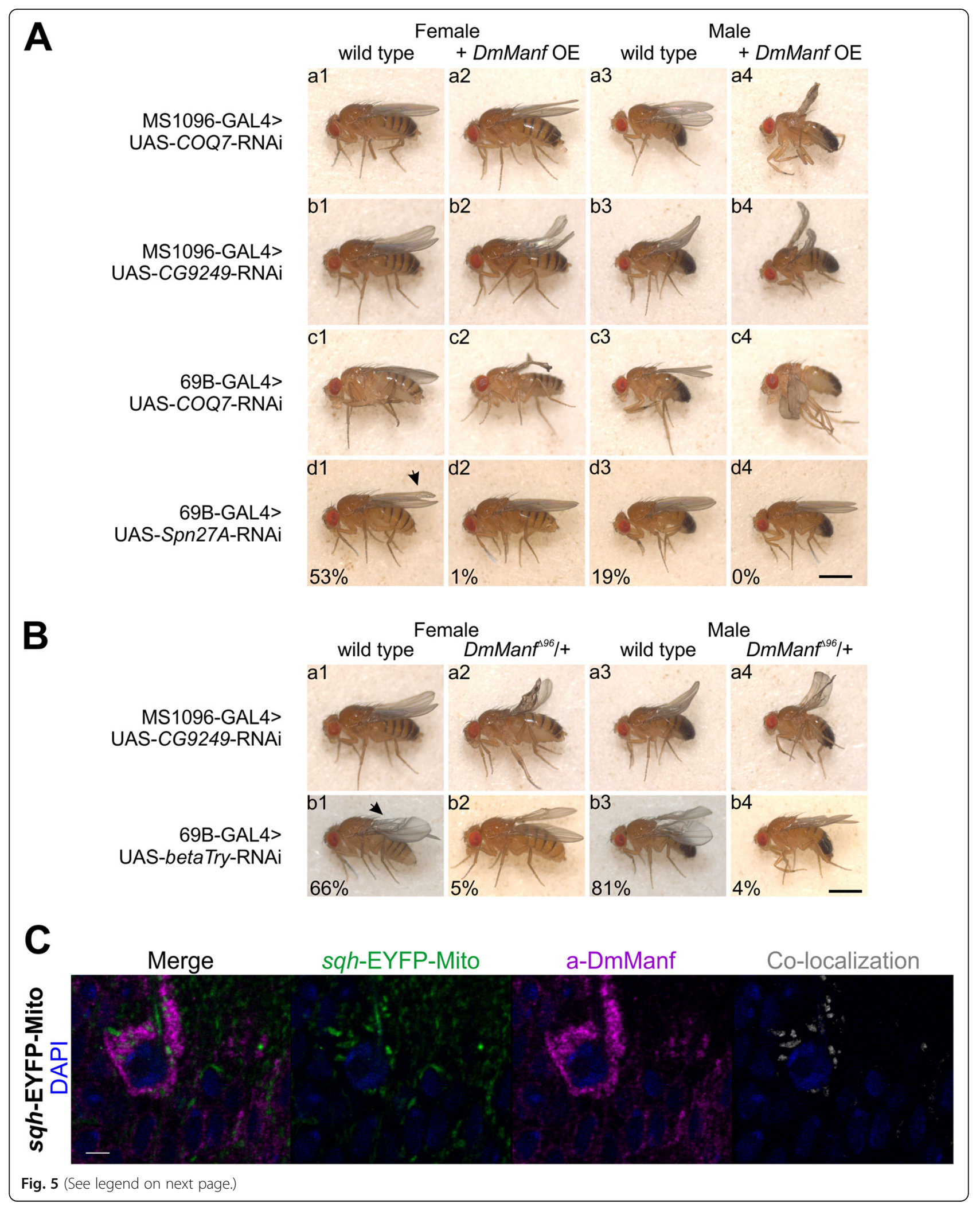


(See figure on previous page.)

Fig. 5 Knockdown of candidate genes is affected by genetically altered DmManf level. Overexpression of DmManf (a) and heterozygous DmManf ${ }^{\Delta 96}$ mutant background (b) affect phenotypes of candidate gene knockdown with wing driver MS1096-GAL4 (a and b in $\mathbf{a}$, a in b) and semi-ubiquitous driver 69B-GAL4 ( $c$ and $d$ in $\mathbf{a}$, b in b). The knockdown of COQ7 and CG9249 are discussed in the main text. Knockdown of Spn27A (Serpin-27A, an inhibitor of serine-type endopeptidase activity) with 69B-GAL4 resulted in distal blister phenotype (arrow) with incomplete penetrance ( $\mathrm{d} 1$ and $\mathrm{d} 3 \mathrm{in} \mathbf{a}$ ). When DmManf was simultaneously overexpressed, the prevalence of blistered phenotype was significantly decreased ( $\mathrm{d} 2 \mathrm{and}$ d4 in a and data not shown). Knockdown of betaTry (betaTrypsin, a serine-type endopeptidase involved in proteolysis) with 69B-GAL4 caused a blistered wing phenotype (arrow) with incomplete penetrance (b1 and b3 in b). The heterozygous DmManA ${ }^{\Delta 96}$ mutant background significantly suppressed the prevalence of this blistered wing phenotype (b2 and b4 in $\mathbf{b}$ and data not shown). The percentages in $\mathrm{d} 1-\mathrm{d} 4 \mathrm{in} \mathrm{A}$ and b1-b4 in B represent the proportion of adult flies with indicated phenotype. Number of analysed adults in (a): d1, 75; d2, 127; d3, 63; d4, 142; and in (b): b1, 325; b2, 189; b3, 286; b4, 236. Scale bar $1 \mathrm{~mm}$. OE, overexpression. c Anti-DmManf (magenta) partially co-localized with mitochondrial marker sqh-EYFP-Mito (green) in the thoracic CNS of 3rd instar larvae. Images consist of four laser confocal sections. Nuclei are shown in blue and gray represents the co-localization of anti-DmManf and sqh-EYFPMito. See Additional file 5 for 3D volume rendering. Scale bar $3 \mu \mathrm{m}$

overexpressing backgrounds, the decreased Irbp mRNA level was not affected (Fig. 7e) indicating that the overexpression of DmManf does not neither induce nor repress Irbp mRNA expression. We also studied the effect of Irbp knockdown on DmManf protein expression by investigating wing discs of Irbp knockdown 3rd instar larvae. In line with our qPCR analysis, DmManf immunoreactivity was not altered in Irbp knockdown with MS1096-GAL4 in comparison to wild type (Fig. 7b).

Based on the structural homology between the Bax-inhibiting SAP domain of Ku70 and C-terminal domain of MANF [22, 23], we hypothesized that the total loss of DmManf in vivo could lead to decreased inhibition of Bax followed by increased cell death and subsequent DmManf ${ }^{196}$ mutant lethality. Thus, we tested whether the loss of Debcl (death executioner Bcl-2 homologue), a homologue to mammalian proapoptotic Bax subfamily, could rescue the DmManf $f^{196}$ mutant lethality in vivo. We used two debcl mutant alleles (loss-of-function allele $\mathrm{debcl}^{E 26}$ and putative dominant negative allele $d e b c l^{W 105}$ ) to abolish endogenous Debcl [44] and UAS-debcl-RNAi line together with ubiquitous da-GAL4 driver to knock down Debcl. However, neither the loss nor silencing of Debcl could rescue the DmManf ${ }^{196}$ mutant lethality (Additional file 6) suggesting that during development DmManf action through other molecular systems than Debcl is more crucial for viability.

\section{Discussion}

MANF and CDNF form an evolutionarily conserved family of neurotrophic factors [1-3]. Since their discovery, increasing data suggests that these proteins possess other characteristics beyond their neurotrophic properties. The loss of Drosophila homologue, DmManf, results in lethality at early developmental stages with neuronal and cuticular defects [3]. Thus, we wanted to explore

Table 2 Enrichment of Drosophila GO terms in the partial RNAi screen

\begin{tabular}{|c|c|c|c|c|c|}
\hline GO ID & GO term & Genes & Count & Total & P-Value \\
\hline GO:0006743 & ubiquinone metabolic process & COQ7, COQ3/CG9249 & 2 & 2 & 0.013 \\
\hline GO:0006733 & $\begin{array}{l}\text { oxidoreduction coenzyme } \\
\text { metabolic process }\end{array}$ & COQ7, COQ3/CG9249 & 2 & 2 & 0.013 \\
\hline GO:0042375 & $\begin{array}{l}\text { quinone cofactor metabolic } \\
\text { process }\end{array}$ & COQ7, COQ3/CG9249 & 2 & 2 & 0.013 \\
\hline GO:0031966 & mitochondrial membrane & COQ7, ND75, Tom20, CG6455 & 4 & 34 & 0.033 \\
\hline GO:0005740 & mitochondrial envelope & COQ7, ND75, Tom20, CG6455 & 4 & 37 & 0.035 \\
\hline GO:0005739 & mitochondrion & $\begin{array}{l}\text { COQ7, Hsp68, Aats-phe, ND75, Tom20, } \\
\text { CG6455 }\end{array}$ & 6 & 106 & 0.035 \\
\hline GO:0031975 & envelope & COQ7, ND75, Tom20, CG6455 & 4 & 47 & 0.058 \\
\hline GO:0031967 & organelle envelope & COQ7, ND75, Tom20, CG6455 & 4 & 47 & 0.058 \\
\hline GO:0044237 & cellular metabolic process & $\begin{array}{l}\text { betaTry, COQ7, dome, Hsp68, Ku70/lrbp, } \\
\text { ND75, Aats-phe, pABp, Spn27A, Taf5, Ts, } \\
\text { Cdk12/CG7597, COQ3/CG9249 }\end{array}$ & 13 & 569 & 0.064 \\
\hline GO:0031090 & organelle membrane & COQ7, ND75, Tom20, CG6455 & 4 & 54 & 0.078 \\
\hline
\end{tabular}

Analysis of Gene Ontology (GO) terms was performed on 21 candidate genes by GOstat (http://gostat.wehi.edu.au/). GO IDs with $P$-value $<0.1$, corresponding GO terms and list of genes are presented. Count, number of our candidate genes mapping to a $\mathrm{GO}$ term; Total, number of genes in our primary screen annotated with each GO term 

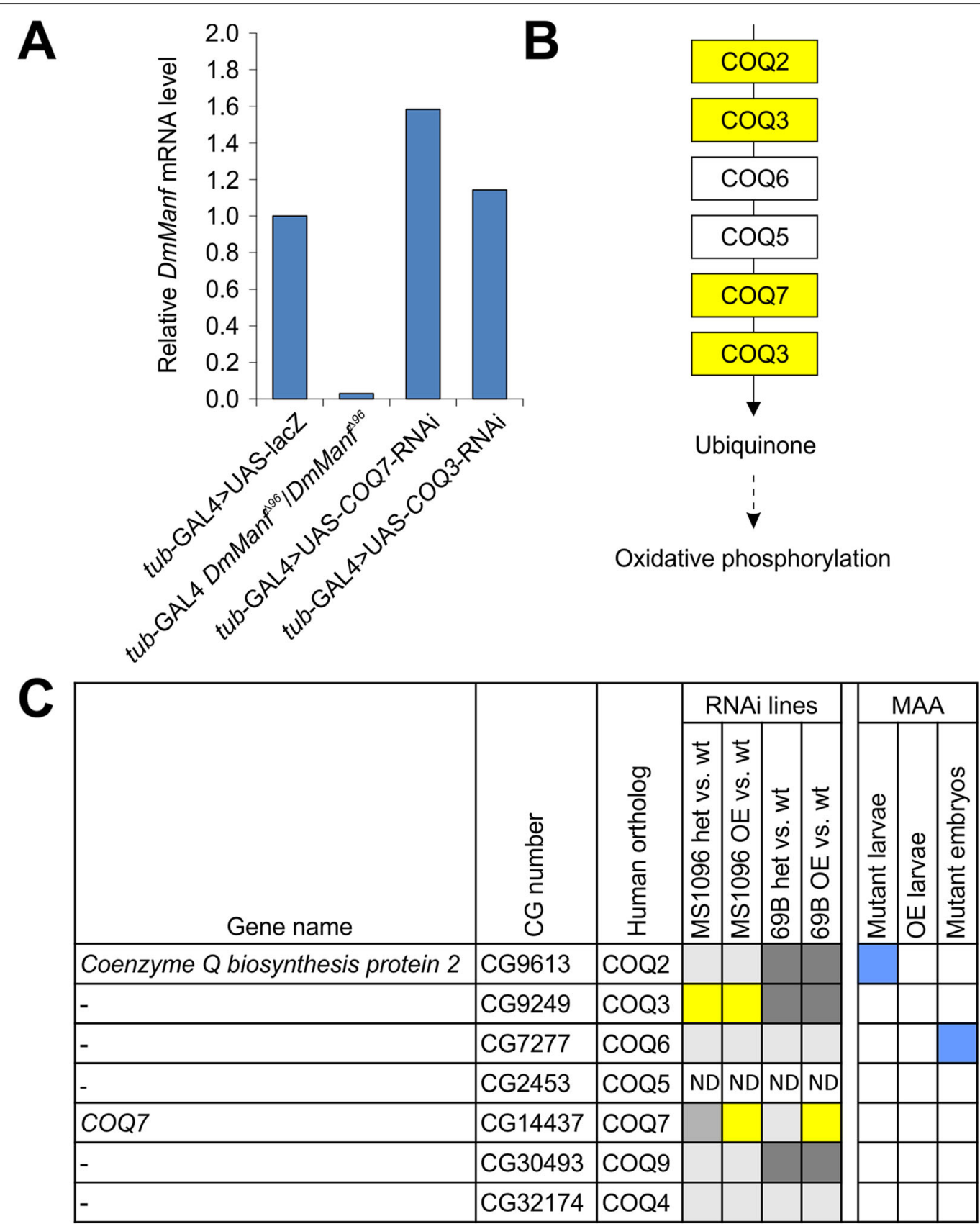

Color key / RNAi lines:

Color key / microarray data:

Stronger phenotype

No phenotype in either genotypes

Downregulated
Not found

Phenotypes similar in both genotypes

Lethal phenotype in both genotypes

Fig. 6 DmManf interacts with genes involved in the ubiquinone synthesis pathway. a Quantitative RT-PCR analysis showed increased DmManf mRNA level when COQ7 was ubiquitously knocked down with tub-GAL4. UAS-lacZ was used as a wild type control. b A schematic presentation of ubiquinone synthesis pathway according to Kyoto Encyclopedia of Genes and Genomes (http://www.genome.jp/kegg/, pathway ID 00130). Genes marked with yellow were found to genetically interact with DmManf. $\mathbf{c} \cup A S-R N A i$ lines of genes involved in ubiquinone synthesis pathway were crossed to MS1096-GAL4 and 69B-GAL4 drivers in wild type, heterozygous DmManf ${ }^{966}$ mutant or DmManf overexpression background. See Fig. 4 legend for interpretation and Additional file 2 for schematic presentation of the crosses. Het, heterozygous DmMant ${ }^{\Delta 96}$ mutant; MAA, microarray analysis; ND, not determined; $\mathrm{OE}$, overexpression 
the tissue-specific effects of knockdown of DmManf using UAS-RNAi approach. In a previous study, neither neuronal nor glial knockdown of DmManf showed any obvious phenotypic effects without the overexpression of Dicer-2, a component of RNAi machinery [45]. Here, we demonstrated that knockdown of DmManf by UASDmManf-RNAi construct is effective and specific. The ubiquitous knockdown resembled the lethal phenotype of homozygous DmManf ${ }^{196}$ mutants. We detected a phenotype in the wing when knocking down DmManf with a wing-specific driver MS1096-GAL4. The wing phenotype was stronger in heterozygous DmManf ${ }^{196}$ mutant background as compared to the wild type background further demonstrating the specificity of UAS-DmManf-RNAi construct. Although the phenotype of small or even absent wings observed in the DmManf knockdown adult males would suggest a decrease in cell number, the number of mitotic cells was significantly increased in the wing disc of DmManf knockdown larvae. Our results are in accordance with the previous study showing that the silencing of human MANF in HeLa cells stimulated cell proliferation [17]. The increased appearance of the mitotic markers used in this study could also be due to prolonged $M$ phase of cell cycle. This would be in line with our finding that the density of cells was not altered between wild type and DmManf knockdown larval wing discs (data not shown). Prolonged cell cycle would result in decreased growth rate and could explain the wing phenotype observed in DmManf knockdown flies. Furthermore, we failed to detect any differences with apoptotic markers (data not shown) indicating that the wing phenotype did not result from increased cell death at the 3rd instar larval stage. However, it is possible that apoptosis could take place later at the pupal stage.

The knowledge on the molecular mechanisms and signaling pathways of MANF/CDNF family proteins is still limited. Previous studies show strong evidence supporting MANF function in ER stress and unfolded protein response $[8,9,15,17,20]$. In our recent study, we showed that the expression of DmManf mRNA is upregulated in response to ER stress-inducing drugs and that DmManf genetically interacts with genes known to function in ER stress and unfolded protein response [13]. In the current work we utilized the wing phenotype detected in DmManf knockdown flies and performed a partial unbiased screen of two genome-wide UAS-RNAi libraries using the Drosophila in vivo model. The primary screen was done with wing-specific driver (MS1096-GAL4) and the selection of candidate genes was based solely on phenotypic similarity to DmManfknockdown flies. We were unable to find genes with ERand ER stress-related functions as potential interaction partners for DmManf because of this criterion: knockdown of ER stress related genes with MS1096GAL4 driver resulted in distinct phenotypes in comparison to the knockdown of DmManf [13]. In the secondary and tertiary screens we knocked down the candidate genes in (1) heterozygous DmManf ${ }^{96}$ mutant background to decrease the level of endogenous DmManf protein and (2) with simultaneous overexpression of DmManf to increase it [3]. Thus, we aimed to discover whether the phenotype caused by silencing of a particular gene would be affected by manipulating DmManf expression level.

Although our screen was only partial, it strongly suggested that DmManf interacts with genes encoding mitochondrial proteins. Mitochondria play crucial roles e.g. in oxidative phosphorylation and $\mathrm{Ca}^{2+}$ signaling, and their dysfunctional biogenesis and metabolism are involved in a variety of human diseases (reviewed in [46-48]). We found that DmManf partially co-localized with the mitochondrial marker sqh-EYFP-Mito. Deeper analysis revealed that strongest DmManf expression was detected adjoining the mitochondrial marker. In sqhEYFP-Mito marker, the fluorophore is directed to mitochondria by the signal peptide of human Cox8A (cytochrome $\mathrm{C}$ oxidase subunit 8A) and localized to the mitochondrial matrix [41]. Since DmManf is also localized to ER [10, 14], the observed co-localization could be on the membranes connecting ER and mitochondria. DmManf could take part in the ER-mitochondrial crosstalk and disturbances in DmManf protein levels could affect protein transport to mitochondria. A specific complex, TOM (translocase of outer membrane), is needed for proper targeting of mitochondrial proteins encoded by nuclear DNA (reviewed in [49]). We identified genetic interaction between DmManf and Tom20 (Translocase of the outer membrane 20, homologue to human TOMM20 and TOMM20-L), a receptor subunit of TOM. Interestingly, another receptor protein of TOM, Maggie/TOMM22 has been shown to mediate localization of pro-apoptotic Debcl (homologue of mammalian Bax) to mitochondria in Drosophila [50]. Bax, together with other members of the Bcl-2 protein family, regulates permeabilization of mitochondrial outer membrane during apoptosis (reviewed in [51]). Previous studies have revealed a role for MANF in Bax-induced cell death in vitro $[10,22]$. In addition, our screen data suggests a genetic interaction between MANF and Ku70/Irbp, an inhibitor of Bax/Debcl. In future, it would be interesting to evaluate whether DmManf co-localizes with TOM proteins and whether Irbp or Debcl have any role in this interaction.

Two genes from the ubiquinone synthesis pathway, COQ7 and CG9249/COQ3, were included in our primary screen and identified as candidate interacting partners of DmManf. We also found evidence for interaction between DmManf and a third component of 


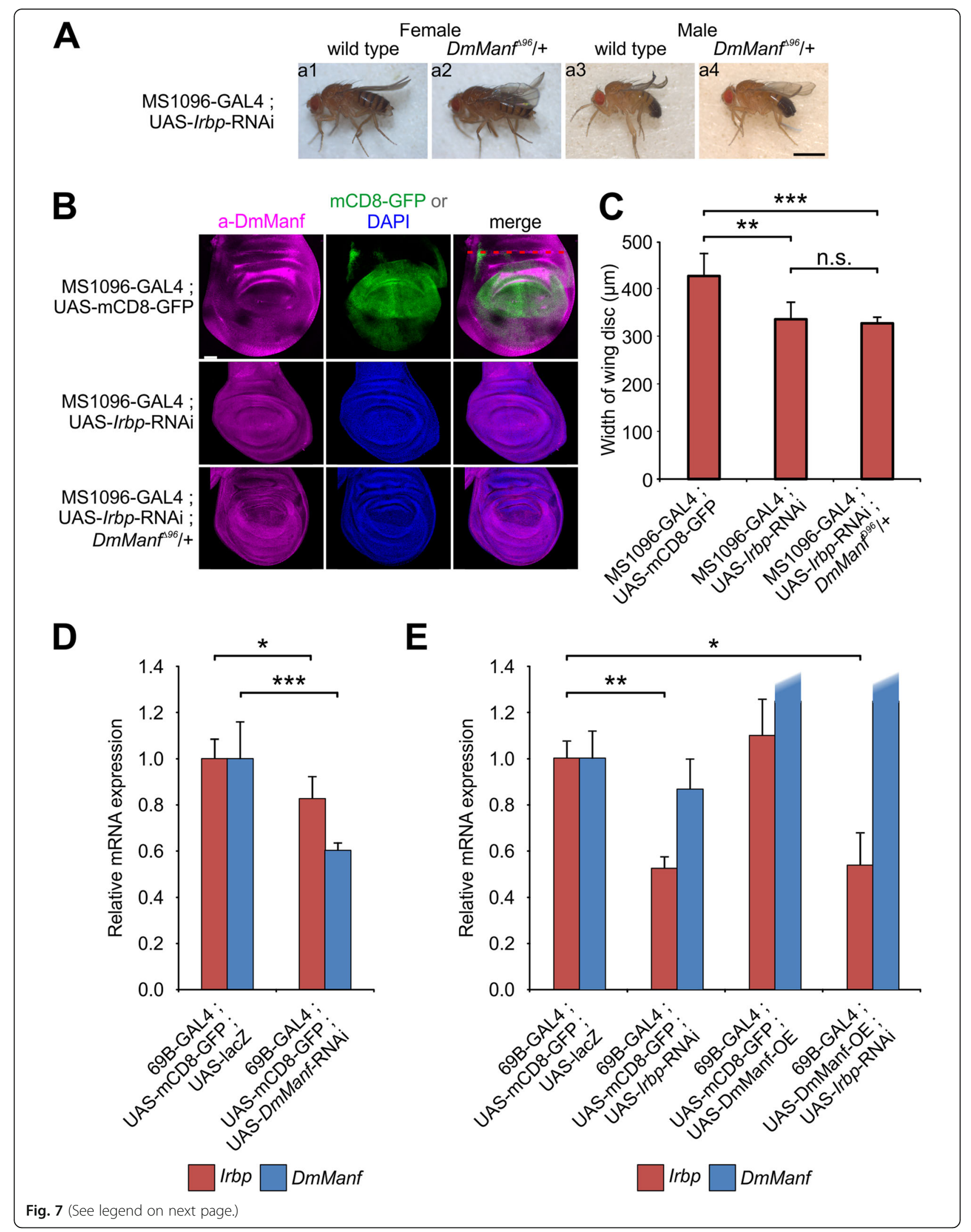


(See figure on previous page.)

Fig. 7 The Drosophila homologue of Ku70, Irbp, is a genetic interacting partner of DmManf. a Heterozygous DmManA ${ }^{\Delta 96}$ mutant background affected phenotype of Irbp knockdown with wing driver MS1096-GAL4 in adult male flies (a3-a4). Scale bar 1 mm. b-c The wing discs of Irbp knockdown with MS1096-GAL4 showed significantly decreased width (red dashed line) in comparison to control genotype (MS1096-GAL4; UAS-mCD8-GFP). $n=8$ (control), $n=5$ (Irbp-RNAi in wild type background), $n=7$ (Irbp-RNAi in heterozygous DmManf ${ }^{\Delta 96}$ mutant background). ${ }^{* *}, P<0.01$, ${ }^{* * *}, P<0.001$, Student's t-test. n.s., not significant. Scale bar 50 m. d-e Knockdown embryos of DmManf with 69B-GAL4 showed decreased DmManf and Irbp mRNA levels (d) and Irbp mRNA expression was decreased in 3rd instar larvae of Irbp knockdown with 69B-GAL4 analysed by qPCR (e). Overexpression of DmManf did not affect Irbp mRNA expression. DmManf mRNA expression was not altered in Irbp knockdown with 69B-GAL4. Overexpression of DmManf with 69B-GAL4 increased DmManf mRNA level (relative mRNA expression $>30$; not shown). ${ }^{*}, P<0.05,{ }^{* *}, P<0.01$, ${ }^{* * *}, P<0.001$, Student's t-test

ubiquinone synthesis pathway, CG9613/COQ2. The knockdown of COQ7 or CG9249/COQ3 resulted in elevated DmManf mRNA levels. The best known function of ubiquinone, also known as coenzyme $Q(Q)$, is its participation in electron transport chain (ETC) by transferring electrons from complexes I and II to complex III (reviewed in [42, 43, 52]). COQ7 hydroxylates demethoxyubiquinone (DMQ) into hydroxyquinone [53] from which ubiquinone is formed by COQ3 [54]. Loss of COQ7 (also known as Mclk-1 in mice, clk-1 in nematodes) leads to Q deficiency and impaired ATP synthesis [55-58]. Q deficiency in humans (OMIM 607426) is associated with variety of clinical manifestations, mostly neuronal and muscular defects (reviewed e.g. in [59]). Importantly, increasing evidence suggests that mitochondrial dysfunction is one of the main causes of PD (reviewed e.g. in [48]) and studies on neuroprotection by $\mathrm{Q}$ treatment in PD models have been promising (reviewed in [60]). Q deficiency has been linked to destabilization of mitochondrial complex I [61]. Complex I is also associated with PD as mutations in its subunits are found to be involved in a familial form of PD (OMIM 556500; reviewed in [48]). Furthermore, toxins used to induce PD-like symptoms in animal models include MPTP, rotenone and paraquat which all interfere with complex I functionality $[62,63]$. In addition to genes involved in Q synthesis, we found homologues for two genes linked to human mitochondrial complex I deficiency (OMIM 252010), ND75 (NDUFS1) and $l(2) 37 B b$ (FOXRED1) to genetically interact with DmManf. Considering all our data indicating a mitochondrial function, DmManf could affect the oxidative phosphorylation, directly or indirectly. In future studies, the connection between DmManf protein and its function in mitochondria should be thoroughly examined.

Alternatively, DmManf could play a role in maintaining cellular $\mathrm{Ca}^{2+}$ homeostasis, an important function of both mitochondria and ER, based on $\mathrm{Ca}^{2+}$-dependent binding of mammalian MANF and GRP78 [8]. In our previous study, cultured Schneider 2 -cells showed a strong induction of DmManf mRNA expression in response to thapsigargin, an inhibitor of ER membraneresident $\mathrm{Ca}^{2+}$ ATPase, which depletes $\mathrm{Ca}^{2+}$ from ER
[13]. Additionally, one of our candidate genes, CG6455, is homologous to human IMMT (inner membrane protein, mitochondrial) predicted with a function in mitochondrial $\mathrm{Ca}^{2+}$ homeostasis.

For several of our candidate genes, e.g. CG9249/COQ3, knockdown with MS1096-GAL4 in both DmManf ${ }^{196}$ heterozygous mutant (with decreased DmManf protein level) and overexpression (with increased DmManf protein level) background resulted in more severe phenotype in comparison to the phenotype observed in wild type background. This suggests that knockdown of certain genes together with the imbalance of DmManf protein level affects overall cellular homeostasis rather than disturbs a putative stoichiometric relationship between DmManf and candidate gene encoded protein levels. Furthermore, while the genetic interaction discovered may represent a physical or biochemical interaction, it might also indicate a secondary effect resulting from involvement of DmManf and candidate gene in the same signaling pathway or biological process.

\section{Conclusions}

This study revealed that DmManf is involved in Drosophila wing development and expanded our knowledge on the role of MANF in the maintenance of cellular homeostasis. Importantly, we discovered novel genetic interacting partners of DmManf and our study suggests that MANF has a role in mitochondrial function. These data help us understand the molecular mechanism of the evolutionarily conserved MANF/CDNF protein family in future studies.

\section{Methods}

\section{Fly strains and antibodies}

Fly stocks and crosses were maintained at $25{ }^{\circ} \mathrm{C}$. The following fly lines were used in the study: $w^{-}$, UASDmManf $^{133}$ (line L3), UAS-DmManf ${ }^{135}$ (line L5) and $D_{\text {DmManf }}{ }^{196} /$ TM6 Tb Sb EYFP [3], UAS-HsMANF ${ }^{\mathrm{L} 2}$ and UAS-HsCDNF ${ }^{\mathrm{L} 1}$ [10], UAS-lacZ [31]. The following lines were obtained from Bloomington Drosophila Stock Center: 69B-GAL4 (\#1774, [31]), A9-GAL4 (\#8761, [38]), da-GAL4 (\#5460, [64]), en2.4-GAL4 ${ }^{\mathrm{e} 16 \mathrm{E}}$ (\#30564, A. Brand \& K. Yoffe, unpublished), MS1096-GAL4 
(\#8860, [37]), salm-GAL4 (\#5818, [65]), Ser-GAL4 (\#6791, [66]), tub-GAL4/TM6 Tb Sb EYFP (\#5138) and UAS-mCD8-GFP (\#5130) [67], debcll26 (\#27342) and

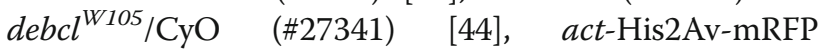
(\#23651, [68]), UAS-GFP.nls (\#4775, [31]) and sqhEYFP-Mito (\#7194, [41]). UAS-S/G2/M-Green was obtained from Kyoto Stock Center (\#109676, [36]). Combination of act-His2Av-mRFP and UAS-S/G2/MGreen in 2nd chromosome was a kind gift from Jinghua Gui. T(2;3)SM6a-TM6B Tb translocation balancer was used in viability studies (referred as SM6-TM6). UASRNAi lines were obtained from Vienna Drosophila RNAi Center and National Institute of Genetics (Additional file 7). Adult flies were imaged with ProgRes SpeedXT camera (Jenoptik). The following antibodies were used: rabbit anti-DmManf [3], rabbit anti-phospho-Histone H3 (Ser10) (06-570, Upstate), anti- $\alpha$-tubulin (DM1A, Sigma).

\section{Immunohistochemistry, confocal microscopy and image analysis}

Third instar larval wing discs and CNS were dissected in PBS and fixed with 4\% paraformaldehyde in PBS or PEM (100 mM PIPES pH 7.0, 2 mM EGTA, $1 \mathrm{mM}$ $\mathrm{MgSO}_{4}$ ) for $30 \mathrm{~min}$. Fixed tissues were washed with PBT (0.1\% Triton X-100 in PBS) and blocked with blocking solution (1\% BSA in PBT) for $1 \mathrm{~h}$. Tissues were incubated with primary antibody overnight at $4{ }^{\circ} \mathrm{C}$ and with secondary antibody for $1 \mathrm{~h}$ in room temperature, and mounted in VECTASHIELD ${ }^{\circ}$ Mounting Medium (Vector Laboratories). Samples were imaged with TCS SP5 laser scanning microscope (Leica Microsystems) equipped with HCX PL APO $20 \times / 0.7 \mathrm{~mm}$ Imm Corr glycerol immersion objective or HC PL APO 10×/0.4 air objective. For co-localization study, Zeiss LSM5 DUO confocal microscope equipped with PL APO $100 \times / 1.4$ oil objective was used. ImageJ 1.43u [69], Imaris 7.6.0 and Imaris 8.4.1 (Bitplane Inc.) were used for image analysis. For quantification of pHis3 positive cells, automatic "Spots" algorithm in Imaris 7.6.0 was used. For quantification of S/G2/M-Green and act-His2Av-RFP positive cells, a $37.9 \mu \mathrm{m} \times 37.9 \mu \mathrm{m}$ area of the dorsal wing pouch was analyzed with the Spots algorithm in Imaris 8.4.1.

\section{Western blot analysis}

Lysis buffer $(20 \mathrm{mM}$ Tris- $\mathrm{HCl} \mathrm{pH}$ 7.4, $150 \mathrm{mM} \mathrm{NaCl}$, 1\% Triton X-100, $1 \mathrm{mM}$ EDTA) supplemented with Complete proteinase inhibitor tablets (Roche) was used in homogenization of larvae. Western blotting was done according to manufacturer's instructions and visualized by the Odyssey infrared imager (Li-Cor).

\section{Adult wing preparations}

Adult flies were dipped into $70 \%$ ethanol and fixed $10 \mathrm{~min}$ in clove oil (Sigma). Wings were dissected and mounted in $70 \%$ Canada Balsam $/ 30 \%$ xylene. Nikon SMZ1500 was used for imaging.

\section{Quantitative RT-PCR}

Larvae were grown at $25{ }^{\circ} \mathrm{C}$ on apple juice plates and collected 50-54 h after egg laying. For 3rd instar larval samples wandering larvae were collected from the vials. Embryos were collected from apple juice plates 16-22 h after egg laying. NucleoSpin ${ }^{\circ}$ RNA II (Macherey-Nagel) was used in extraction and purification of total RNA. DNase treatment was done on-column according to the manufacturer's instructions. First strand cDNA was synthesized from total RNA $(1 \mu \mathrm{g})$ using RevertAid Premium Reverse Transcriptase (Thermo Scientific) and Oligo $\left(\mathrm{dT}_{18}\right)$ primer at $53{ }^{\circ} \mathrm{C}$ according to manufacturer's instructions. Expression of DmManf mRNA was quantified by LightCycler 480 Real-Time PCR System with Lightcycler 480 SYBR Green I master mix (Roche) with primers DmManf forward 5'-AATCTGCGACCTTCG CTATG-3'and DmManf reverse 5'-TCGTTGAGGAT TTTCTTCAGG-3' [14]. Irbp was amplified with primers Irbp forward 5'-AGTTCATCACGTTGTCAA GAGC-3' and Irbp reverse 5'-TACGATCGGACAGG ATTTCG-3' [70]. RpL32 was amplified as a reference gene with primers RpL32 forward 5'-CGGATCGATA TGCTAAGCTGT-3' and RpL32 reverse 5'-GCGC TTGTTCGATCCGTA-3' [14]. PCR efficiency (E) of each primer pair was determined from a relative standard curve. For DmManf, $\mathrm{E}=1.98$; for $\operatorname{Irbp}, \mathrm{E}=2.00$; for $R p L 32, \mathrm{E}=1.97$. Equation $\mathrm{E}^{-\mathrm{Cp}}$ in which $\mathrm{Cp}$ indicates a crossing point was used to calculate relative concentration of DmManf, Irbp and RpL32 mRNA in each sample. To present the results, the concentration of DmManf and Irbp was normalized to the level of RpL32. Each sample was analysed as a duplicate.

\section{Statistical analysis}

Means were compared by Student's t-test, null hypothesis was rejected at $P<0.05$. Statistical analyses were performed by using Microsoft ${ }^{\circ}$ Excel Analysis ToolPak (Microsoft ${ }^{\circ}$ Office Professional Plus 2010). For pupal viability studies, normal $\left(\right.$ Tubby $\left.{ }^{+} ; \mathrm{Tb}^{+}\right)$and squat $\left(\mathrm{Tb}^{-}\right)$ pupae were counted, the number of $\mathrm{Tb}^{+}$pupae was divided by the number of all pupae and normalized to experimentally determined ratio from tub-GAL4/TM6 Tb $\mathrm{Sb}$ cross to wild type and to wild type balanced against SM6-TM6 translocation balancer (Additional file 4 and Additional file 8, wild type and DmManf overexpression data previously reported in [13]). For preliminary analyses 
two vials were counted and statistical analysis was done based on six vials with minimum of 40 pupae. For genotypes showing incomplete penetrance of the wing phenotypes, quantification of the penetrance was performed by counting adult flies with and without phenotype from 4 vials per genotype.

\section{Gene ontology analysis}

Gene Ontology (GO) analysis was performed for genes considered as hits from our UAS-RNAi screen (21 genes) against the set of genes included in the primary screen (approximately 2800 randomly selected genes). GOstat (http://gostat.wehi.edu.au/) was used with default tool settings. A complete list of overrepresented $(p<0.1)$ GO terms is presented in Table 2.

\section{Additional files}

Additional file 1: Two DmManf-RNAi constructs in three independent transformant lines are available in VDRC. A tiff file. A) Alignment of DmManfRNAi constructs 4793 and 108792. Construct 4793 targets last three exons of the DmManf gene with no predicted off-targets (VDRC data sheet). Construct 108792 targets exon 4 and 3' UTR and has one predicted offtarget (Sulfated/CG6725; VDRC data sheet). Construct 4793 in transformant line 12835 was used in further studies. B) Different UAS-DmManf-RNAi transformant lines show similar phenotypes when driven with wing-specific MS1096-GAL4. For all lines, heterozygous DmMan ${ }^{\Delta 96}$ mutant background lead to stronger wing phenotype and overexpression (OE) of DmManf rescued the wing phenotype. C) Alignment of UAS-DmManf-RNAi construct (ID 4793) with UAS-HSMANF and UAS-HSCDNF constructs. Strongest alignment is shown in green (HsMANF) and purple (HsCDNF). VDRC, Vienna Drosophila RNAi Center; Tf, transformant line; OE, overexpression. (TIFF $2536 \mathrm{~kb}$ )

Additional file 2: Screen for genetic interaction partners of DmManf. A tiff file. A) Scheme of the crosses used in the partial RNAi library screen. First, randomly selected UAS-x-RNAi lines were crossed to wing-specific driver line MS1096-GAL4 (1). Lines showing similar phenotype to UAS-DmManf-RNAi lines were selected to the next stage. Second, two GAL4 drivers, wing-specific MS1096-GAL4 and semi-ubiquitous 69B-GAL4, were used in both wild type and heterozygous DmMant ${ }^{\Delta 96}$ mutant background (2) - UAS-x-RNAi lines showing distinct phenotypes in wild type and heterozygous DmMant ${ }^{\Delta 96}$ mutant backgrounds were selected. Secondary stage was repeated for selected UAS-x-RNAi lines in order to ensure the observed interactions. At stage 3, 69B-GAL4 and MS1096-GAL4 drivers were used to express UAS-X-RNAi lines with or without UAS-DmManf overexpression construct (3). Based on stages 2 and 3, 21 UAS-x-RNAi lines were selected as candidates for final stage 4. (4) UAS-x-RNAi lines were expressed with tub-GAL4 with and without DmManf overexpression to study whether high levels of DmManf affected ubiquitous silencing of selected genes (see Additional file 4). B-C) DmManf (magenta) was ubiquitously expressed in the wing disc of 3rd instar larvae. MS1096-GAL4 expression detected by UAS-mCD8-GFP (green) was found mainly in the dorsal wing compartment but also in other regions of the wing disc (B). MS1096-GAL4 expression pattern was also detected in the CNS (C). Nuclear counterstain DAPI (gray) was used to mark the tissue morphology. Scale bar $50 \mu \mathrm{m}$. D) Insertion of GAL4 construct GawB in driver lines 69B-GAL4 and MS1096-GAL4 [31] did not affect adult fly phenotype (+/+, top row). Heterozygous DmManf mutation (DmMan ${ }^{\Delta 96} /+$, middle row) or overexpression of DmManf (UAS-DmManf $f^{5}$, bottom row) together with 69B-GAL4 and MS1096-GAL4 insertions showed no obvious phenotype in adult flies. (TIFF $3486 \mathrm{~kb}$ )

Additional file 3: Examples of candidate genes for interacting partners of DmManf. A tiff file. A) MS1096-GAL4 was used to silence candidate genes in wild type, heterozygous DmMant ${ }^{\Delta 96}$ mutant $\left(D m M a n A^{\Delta 96} /+\right)$ and
DmManf overexpression (+ DmManf OE) backgrounds. With many candidate genes, both heterozygous DmManf ${ }^{\Delta 96}$ mutant background and DmManf overexpression resulted in more severe phenotype. For example, knockdown of Tom20 (Translocase of outer membrane 20, a mitochondrial transmembrane transporter protein) showed a mildly wrinkled wing phenotype in wild type background (a1). When heterozygous DmMan ${ }^{\Delta 96}$ mutant background (a2) or simultaneous DmManf overexpression was used (a3), wings were strongly wrinkled. Similarly, knockdown of Cdk12 (a cyclin-dependent protein serine/threonine kinase; b1-b3) showed stronger phenotype both in heterozygous DmMant ${ }^{\Delta 96}$ mutant and DmManf-overexpressing backgrounds. Complete list of the alterations is presented in Fig. 4. B) Quantitative analysis of ubiquitous knockdown of candidate genes CSN3, Irbp, COQ7 and ubiquinone synthesis related gene COQ2 showed altered pupal viability with DmManf overexpression (+ DmManf $\mathrm{OE}$, red) in comparison to wild type background (- DmManf OE, blue). tub-GAL4/+ flies were used as wild type control. C) Proportion of emerged adults when candidate genes were ubiquitously knocked down with tub-GAL4 with (+ $\mathrm{OE})$ or without (- OE) DmManf overexpression. ${ }^{*}, P<0.05$; ***

$P<0.001$, Student's t-test. Amount of pupae and adults analysed in $\mathrm{B}-\mathrm{C}$ are presented in Additional file 4. Proportion of $\mathrm{Tb}^{+}$pupae was normalized to experimentally determined proportion of $\mathrm{Tb}^{+}$pupae (see Additional file 8, wild type and wild type/SM6-TM6). (TIFF $1442 \mathrm{~kb}$ )

Additional file 4: Results from ubiquitous knockdown studies of UAS-RNAi lines with and without DmManf overexpression. A pdf file. (PDF $10 \mathrm{~kb}$ )

Additional file 5: Video of 3D volume rendering of co-localization of aDmManf and sqh-EYFP-Mito. A mov file. DmManf expression (magenta) adjoined the sqh-EYFP-Mito marker (green). (MOV $6408 \mathrm{~kb}$ )

Additional file 6: Number of heterozygous pupae in the rescue experiments of DmMann ${ }^{\Delta 96}$ mutant lethality by abolishment of debcl. A pdf file. (PDF $29 \mathrm{~kb}$ )

Additional file 7: List of UAS-RNAi lines used in the study. A pdf file. (PDF $5 \mathrm{~kb}$ )

Additional file 8: Results from ubiquitous knockdown of UAS-DmManfRNAi. A pdf file. (PDF $18 \mathrm{~kb}$ )

\section{Acknowledgements}

We are grateful to Evely Vridolin and Osamu Shimmi for performing the majority of the primary screening of UAS-RNAi lines and sharing the results with us. Arja Ikävalko and Sari Tynkkynen are acknowledged for their excellent technical assistance and Zeng Zhao and Lin Feng for help with primary screening. We thank Jukka Kallijärvi and Osamu Shimmi for critical reading of the manuscript and Ville Hietakangas, Shinya Matsuda, Mika Molin and Jaana Vulli for invaluable advice. We thank Bloomington Drosophila Stock Center, Vienna Drosophila RNAi Center, Kyoto Stock Center, National Institute of Genetics (Japan), Osamu Shimmi, Ville Hietakangas and Jinghua Gui for fly stocks, and Juha Partanen for sharing antibodies. Confocal imaging was performed at the Light Microscopy Unit, Institute of Biotechnology.

\section{Funding}

RL was supported by Viikki Doctoral Programme in Molecular Biosciences, The Finnish Parkinson Foundation, The Ella and Georg Ehrnrooth Foundation, The University of Helsinki Funds, and Alfred Kordelin Foundation. PL was supported by The Academy of Finland (grant No. 139910). MP was supported by Estonian Research Council (grant No. IUT19-18). TIH was supported by Helsinki University Funds.

Availability of data and materials

All data generated or analysed during this study are included in this published article and its additional files.

\section{Authors' contributions}

$\mathrm{RL}$ and $\mathrm{TIH}$ designed the research, RL with the help of MP and TIH performed the genetic crosses, immunohistochemistry, microscopy and image analysis, PL performed GPCR and Western blot analyses, RL analysed the data and wrote the draft manuscript. All authors read, made corrections and approved the manuscript.

\section{Competing interests}

The authors declare that they have no competing interests. 


\section{Consent for publication}

Not applicable.

\section{Ethics approval}

Not applicable.

\section{Publisher's Note}

Springer Nature remains neutral with regard to jurisdictional claims in published maps and institutional affiliations.

\section{Author details}

'Department of Biosciences, University of Helsinki, Fl-00014 Helsinki, Finland. ${ }^{2}$ Institute of Biotechnology, University of Helsinki, Fl-00014 Helsinki, Finland. ${ }^{3}$ Department of Chemistry and Biotechnology, Tallinn University of Technology, EE-12618 Tallinn, Estonia. ${ }^{4}$ Current affiliation: Institute of Biotechnology, University of Helsinki, Helsinki, Finland.

Received: 1 September 2016 Accepted: 8 May 2017

Published online: 02 June 2017

\section{References}

1. Petrova PS, Raibekas A, Pevsner J, Vigo N, Anafi M, Moore MK, Peaire AE, Shridhar V, Smith DI, Kelly J, Durocher Y, Commissiong JW. MANF: a new mesencephalic, astrocyte-derived neurotrophic factor with selectivity for dopaminergic neurons. J Mol Neurosci. 2003;20:173-87.

2. Lindholm $\mathrm{P}$, Voutilainen $\mathrm{MH}$, Laurén J, Peränen J, Leppänen V-M, Andressoo J-O, Lindahl M, Janhunen S, Kalkkinen N, Timmusk T, Tuominen RK, Saarma M. Novel neurotrophic factor CDNF protects and rescues midbrain dopamine neurons in vivo. Nature. 2007;448:73-7.

3. Palgi M, Lindström R, Peränen J, Piepponen TP, Saarma M, Heino TI. Evidence that DmMANF is an invertebrate neurotrophic factor supporting dopaminergic neurons. Proc Natl Acad Sci U S A. 2009;106:2429-34.

4. Lindholm P, Saarma M. Novel CDNF/MANF family of neurotrophic factors. Dev Neurobiol. 2010;70:360-71.

5. Voutilainen $M H$, Bäck S, Pörsti E, Toppinen L, Lindgren L, Lindholm $P$, Peränen J, Saarma M, Tuominen RK. Mesencephalic astrocyte-derived neurotrophic factor is neurorestorative in rat model of Parkinson's disease. J Neurosci. 2009;29:9651-9.

6. Airavaara M, Harvey BK, Voutilainen $M H$, Shen $H$, Chou J, Lindholm $P$, Lindahl M, Tuominen RK, Saarma M, Hoffer B, Wang Y. CDNF protects the nigrostriatal dopamine system and promotes recovery after MPTP treatment in mice. Cell Transplant. 2012;21:1213-23.

7. Airavaara M, Chiocco MJ, Howard DB, Zuchowski KL, Peränen J, Liu C, Fang S, Hoffer BJ, Wang Y, Harvey BK. Widespread cortical expression of MANF by AAV serotype 7: localization and protection against ischemic brain injury. Exp Neurol. 2010;225:104-13.

8. Glembotski CC, Thuerauf DJ, Huang C, Vekich JA, Gottlieb RA, Doroudgar S. Mesencephalic astrocyte-derived neurotrophic factor protects the heart from ischemic damage and is selectively secreted upon sarco/endoplasmic reticulum calcium depletion. J Biol Chem. 2012;287:25893-904.

9. Lindahl M, Danilova T, Palm E, Lindholm P, Võikar V, Hakonen E, Ustinov J, Andressoo J-O, Harvey B, Otonkoski T, Rossi J, Saarma M. MANF is indispensable for the proliferation and survival of pancreatic $B$ cells. Cell Rep. 2014;7:366-75.

10. Lindström R, Lindholm P, Kallijärvi J, Yu L-Y, Piepponen TP, Arumäe U, Saarma $M$, Heino TI. Characterization of the structural and functional determinants of MANF/CDNF in Drosophila in vivo model. PLoS One. 2013;8:e73928.

11. Henderson MJ, Richie CT, Airavaara M, Wang Y, Harvey BK. Mesencephalic astrocyte-derived neurotrophic factor (MANF) secretion and cell surface binding are modulated by KDEL receptors. J Biol Chem. 2013;288:4209-25.

12. Chen $L$, Wan $L$, Du J, Shen $Y$. Identification of MANF as a protein interacting with RTN1-C. Acta Biochim Biophys Sin. 2015;47:91-7.

13. Lindström R, Lindholm P, Kallijärvi J, Palgi M, Saarma M, Heino TI. Exploring the conserved role of MANF in the unfolded protein response in Drosophila melanogaster. PLoS One. 2016;11:e0151550.

14. Palgi M, Greco D, Lindström R, Auvinen P, Heino TI. Gene expression analysis of Drosophila Manf mutants reveals perturbations in membrane traffic and major metabolic changes. BMC Genomics. 2012;13:134

15. Mizobuchi N, Hoseki J, Kubota H, Toyokuni S, Nozaki J-J, Naitoh M, Koizumi A, Nagata K. ARMET is a soluble ER protein induced by the unfolded protein response via ERSE-II element. Cell Struct Funct. 2007;32:41-50.
16. Tadimalla A, Belmont PJ, Thuerauf DJ, Glassy MS, Martindale JJ, Gude N, Sussman MA, Glembotski CC. Mesencephalic astrocyte-derived neurotrophic factor is an ischemia-inducible secreted endoplasmic reticulum stress response protein in the heart. Circ Res. 2008;103:1249-58.

17. Apostolou A, Shen Y, Liang Y, Luo J, Fang S. Armet, a UPR-upregulated protein, inhibits cell proliferation and ER stress-induced cell death. Exp Cell Res. 2008;314:2454-67.

18. Lee A-H, Iwakoshi NN, Glimcher LH. XBP-1 regulates a subset of endoplasmic reticulum resident chaperone genes in the unfolded protein response. Mol Cell Biol. 2003;23:7448-59.

19. Girardot F, Monnier V, Tricoire H. Genome wide analysis of common and specific stress responses in adult Drosophila melanogaster. BMC Genomics. 2004:5:74.

20. Nundlall S, Rajpar MH, Bell PA, Clowes C, Zeeff LAH, Gardner B, Thornton DJ, Boot-Handford RP, Briggs MD. An unfolded protein response is the initial cellular response to the expression of mutant matrilin-3 in a mouse model of multiple epiphyseal dysplasia. Cell Stress Chaperones. 2010;15:835-49.

21. Hartley CL, Edwards S, Mullan L, Bell PA, Fresquet M, Boot-Handford RP, Briggs MD. Armet/Manf and Creld2 are components of a specialized ER stress response provoked by inappropriate formation of disulphide bonds: implications for genetic skeletal diseases. Hum Mol Genet. 2013;22:5262-75.

22. Hellman M, Arumäe U, Yu L-Y, Lindholm P, Peränen J, Saarma M, Permi P. Mesencephalic astrocyte-derived neurotrophic factor (MANF) has a unique mechanism to rescue apoptotic neurons. J Biol Chem. 2011;286:2675-80.

23. Sawada M, Hayes P, Matsuyama S. Cytoprotective membrane-permeable peptides designed from the Bax-binding domain of Ku70. Nature Cell Biol. 2003:5:352-7.

24. Cheng L, Zhao H, Zhang W, Liu B, Liu Y, Guo Y, Nie L. Overexpression of conserved dopamine neurotrophic factor (CDNF) in astrocytes alleviates endoplasmic reticulum stress-induced cell damage and inflammatory cytokine secretion. Biochem Biophys Res Commun. 2013;435:34-9.

25. Zhao H, Liu Y, Cheng L, Liu B, Zhang W, Guo Y-J, Nie L. Mesencephalic astrocyte-derived neurotrophic factor inhibits oxygen-glucose deprivationinduced cell damage and inflammation by suppressing endoplasmic reticulum stress in rat primary astrocytes. J Mol Neurosci. 2013;51:671-8.

26. Zhao H, Cheng L, Liu Y, Zhang W, Maharjan S, Cui Z, Wang X, Tang D, Nie L. Mechanisms of anti-inflammatory property of conserved dopamine neurotrophic factor: inhibition of JNK signaling in lipopolysaccharideinduced microglia. J Mol Neurosci. 2014;52:186-92.

27. Nadella R, Voutilainen MH, Saarma M, Gonzalez-Barrios JA, Leon-Chavez BA, Jimnez JMD, Jimnez SHD, Escobedo L, Martinez-Fong D. Transient transfection of human CDNF gene reduces the 6-hydroxydopamine-induced neuroinflammation in the rat substantia nigra. J Neuroinflamm. 2014;11:209.

28. Chen L, Feng L, Wang X, Du J, Chen Y, Yang W, Zhou C, Cheng L, Shen Y, Fang S, Li J, Shen Y. Mesencephalic Astrocyte-derived Neurotrophic factor is involved in inflammation by negatively regulating the NF-KB pathway. Sci Rep. 2015:5:8133.

29. Hoesel B, Schmid JA. The complexity of NF-KB signaling in inflammation and cancer. Mol Cancer. 2013;12:86.

30. Neves J, Zhu J, Sousa-Victor P, Konjikusic M, Riley R, Chew S, Qi Y, Jasper H, Lamba DA. Immune modulation by MANF promotes tissue repair and regenerative success in the retina. Science. 2016;353:6294.

31. Brand $\mathrm{AH}$, Perrimon N. Targeted gene expression as a means of altering cell fates and generating dominant phenotypes. Development. 1993;118:401-15.

32. Fire A, Xu S, Montgomery MK, Kostas SA, Driver SE, Mello CC. Potent and specific genetic interference by double-stranded RNA in Caenorhabditis elegans. Nature. 1998;391:806-11.

33. Dietzl G, Chen D, Schnorrer F, Su K-C, Barinova Y, Fellner M, Gasser B, Kinsey K, Oppel S, Scheiblauer S, Couto A, Marra V, Keleman K, Dickson BJ. A genome-wide transgenic RNAi library for conditional gene inactivation in Drosophila. Nature. 2007:448:151-6.

34. Hendzel MJ, Wei Y, Mancini MA, Van Hooser A, Ranalli T, Brinkley BR, BazettJones DP, Allis CD. Mitosis-specific phosphorylation of histone $\mathrm{H} 3$ initiates primarily within pericentromeric heterochromatin during $\mathrm{G} 2$ and spreads in an ordered fashion coincident with mitotic chromosome condensation. Chromosoma. 1997;106:348-60

35. Sakaue-Sawano A, Kurokawa H, Morimura T, Hanyu A, Hama H, Osawa H, Kashiwagi S, Fukami K, Miyata T, Miyoshi H, Imamura T, Ogawa M, Masai H, Miyawaki A. Visualizing spatiotemporal dynamics of multicellular cell-cycle progression. Cell. 2008;132:487-98. 
36. Nakajima Y, Kuranaga E, Sugimura K, Miyawaki A, Miura M. Nonautonomous apoptosis is triggered by local cell cycle progression during epithelial replacement in Drosophila. Mol Cell Biol. 2011;31:2499-512.

37. Capdevila J, Guerrero I. Targeted expression of the signaling molecule decapentaplegic induces pattern duplications and growth alterations in Drosophila wings. EMBO J. 1994;13:4459-68.

38. Lin DM, Goodman CS. Ectopic and increased expression of fasciclin II alters motoneuron growth cone guidance. Neuron. 1994;13:507-23.

39. Neumann CJ, Cohen SM. Distinct mitogenic and cell fate specification functions of wingless in different regions of the wing. Development. 1996;122:1781-9.

40. McQuilton P, St Pierre SE, Thurmond J, Gelbart W, Brown N, Kaufman T, Matthews K, Werner-Washburne M, Cripps R, Crosby L, Dirkmaat A, Emmert D, Gramates LS, Falls K, Matthews B, Russo S, Schroeder A, St Pierre S, Zhou P, Zytkovicz M, Adryan B, Bunt S, Costa M, Field H, Marygold S, Millburn G, Ponting L, Osumi-Sutherland D, Stefancsik R, Tweedie S, Atrill H, Goodman J, Grumbling G, Strelets V, Wong JD, Platero H. FlyBase 101 - the basics of navigating FlyBase. Nucleic Acids Res. 2012;40:D706-14.

41. LaJeunesse DR, Buckner SM, Lake J, Na C, Pirt A, Fromson K. Three new Drosophila markers of intracellular membranes. BioTechniques. 2004;36:784-90.

42. Hatefi Y. The mitochondrial electron transport and oxidative phosphorylation system. Annu Rev Biochem. 1985;54:1015-69.

43. Crane FL: Discovery of ubiquinone (coenzyme Q) and an overview of function. Mitochondrion 2007;7(Suppl):S2-S7.

44. Sevrioukov EA, Burr J, Huang EW, Assi HH, Monserrate JP, Purves DC, Wu JN, Song EJ, Brachmann CB. Drosophila BCl-2 proteins participate in stressinduced apoptosis, but are not required for normal development. Genesis. 2007;45:184-93.

45. Stratoulias V, Heino TI. Analysis of the conserved neurotrophic factor MANF in the Drosophila adult brain. Gene Expr Patterns. 2015;18:8-15.

46. Scharfe C, Zaccaria P, Hoertnagel K, Jaksch M, Klopstock T, Lill R, Prokisch H, Gerbitz K-D, Mewes HW, Meitinger T. MITOP: database for mitochondriarelated proteins, genes and diseases. Nucleic Acids Res. 1999;27:153-5.

47. Balaban RS, Nemoto S, Finkel T. Mitochondria, oxidants, and aging. Cell. 2005;120:483-95.

48. Schon E, Przedborski S. Mitochondria: the next (Neurode)generation. Neuron. 2011;70:1033-53.

49. Schatz G. The protein import system of mitochondria. J Biol Chem. 1996; 271:31763-6.

50. Colin J, Garibal J, Mignotte B, Guénal I. The mitochondrial TOM complex modulates bax-induced apoptosis in Drosophila. Biochem Biophys Res Commun. 2009;379:939-43

51. Chipuk JE, Green DR. How do BCL-2 proteins induce mitochondrial outer membrane permeabilization? Trends Cell Biol. 2008;18:157-64.

52. Aguilaniu H, Durieux J, Dillin A. Metabolism, ubiquinone synthesis, and longevity. Genes Dev. 2005;19:2399-406.

53. Marbois BN, Clarke CF. The COQ7 gene encodes a protein in Saccharomyces cerevisiae necessary for ubiquinone biosynthesis. J Biol Chem. 1996;271:2995-3004.

54. Poon WW, Barkovich RJ, Hsu AY, Frankel A, Lee PT, Shepherd JN, Myles DC, Clarke CF. Yeast and rat Coq3 and Escherichia coli UbiG polypeptides catalyze both O-methyltransferase steps in coenzyme Q biosynthesis. J Biol Chem. 1999;274:21665-72.

55. Jonassen T, Proft M, Randez-Gil F, Schultz JR, Marbois BN, Entian K-D, Clarke CF. Yeast clk-1 homologue (Coq7/Cat5) is a mitochondrial protein in coenzyme Q synthesis. J Biol Chem. 1998;273:3351-7.

56. Jonassen $\mathrm{T}$, Larsen PL, Clarke CF. A dietary source of coenzyme Q is essential for growth of long-lived Caenorhabditis elegans clk-1 mutants. Proc Natl Acad Sci U S A. 2001:98:421-6.

57. Stenmark P, Grünler J, Mattsson J, Sindelar PJ, Nordlund P, Berthold DA. A new member of the family of di-iron carboxylate proteins. Coq7 (clk-1), a membrane-bound hydroxylase involved in ubiquinone biosynthesis. J Biol Chem. 2001;276:33297-300.

58. Takahashi M, Shimizu T, Shirasawa T. Reversal of slow growth and heartbeat through the restoration of mitochondrial function in clk-1-deficient mouse embryos by exogenous administration of coenzyme Q 10. Exp Gerontol. 2012;47:425-31.

59. Quinzii CM, López LC, Naini A, Dimauro S, Hirano M. Human CoQ10 deficiencies. Biofactors. 2008;32:113-8.

60. Jin H, Kanthasamy A, Ghosh A, Anantharam V, Kalyanaraman B, Kanthasamy AG. Mitochondria-targeted antioxidants for treatment of Parkinson's disease: preclinical and clinical outcomes. Biochim Biophys Acta. 2014;1842(8):1282-94.
61. García-Corzo L, Luna-Sánchez M, Doerrier C, García JA, Guarás A, Acín-Pérez R, Bullejos-Peregrín J, López A, Escames G, Enríquez JA, Acuña-Castroviejo D, López LC. Dysfunctional coq9 protein causes predominant encephalomyopathy associated with CoQ deficiency. Hum Mol Genet. 2013; 22:1233-48.

62. Langston JW, Ballard P, Tetrud JW, Irwin I. Chronic parkinsonism in humans due to a product of meperidine-analog synthesis. Science. 1983;219:979-80.

63. Betarbet R, Sherer TB, MacKenzie G, Garcia-Osuna M, Panov AV, Greenamyre JT. Chronic systemic pesticide exposure reproduces features of Parkinson's disease. Nat Neurosci. 2000;3:1301-6.

64. Wodarz A, Hinz U, Engelbert M, Knust E. Expression of crumbs confers apical character on plasma membrane domains of ectodermal epithelia of Drosophila. Cell. 1995;82:67-76.

65. Hinz U, Giebel B, Campos-Ortega JA. The basic-helix-loop-helix domain of Drosophila lethal of scute protein is sufficient for proneural function and activates neurogenic genes. Cell. 1994;76:77-87.

66. Fleming RJ, Gu Y, Hukriede NA. Serrate-mediated activation of notch is specifically blocked by the product of the gene fringe in the dorsal compartment of the Drosophila wing imaginal disc. Development. 1997; 124:2973-81.

67. Lee T, Luo L. Mosaic analysis with a repressible neurotechnique cell marker for studies of gene function in neuronal morphogenesis. Neuron. 1999;22: 451-61.

68. Schuh M, Lehner CF, Heidmann S: Incorporation of Drosophila CID/CENP-A and CENP-C into Centromeres during early embryonic anaphase. Curr Biol. 2007:17:237-43.

69. Abràmoff MD, Magalhães PJ, Ram SJ. Image processing with ImageJ. Biophoton Int. 2004;11:36-41.

70. Sopko R, Foos M, Vinayagam A, Zhai B, Binari R, Hu Y, Randklev S, Perkins LA, Gygi SP, Perrimon N. Combining genetic perturbations and proteomics to examine Kinase-Phosphatase networks in Drosophila embryos. Dev Cell. 2014;31:114-27.

\section{Submit your next manuscript to BioMed Central and we will help you at every step:}

- We accept pre-submission inquiries

- Our selector tool helps you to find the most relevant journal

- We provide round the clock customer support

- Convenient online submission

- Thorough peer review

- Inclusion in PubMed and all major indexing services

- Maximum visibility for your research

Submit your manuscript at www.biomedcentral.com/submit

) Biomed Central 